\title{
Microstructural Evolution in Orthotropic Elastic Media
}

\author{
P. H. Leo, ${ }^{*}$ J. S. Lowengrub, $\dagger^{1}$ and Qing Nie $\ddagger^{2}$ \\ * Department of Aerospace Engineering and Mechanics, University of Minnesota, Minneapolis, Minnesota \\ 55455; †School of Mathematics, University of Minnesota, Minneapolis, Minnesota 55455; \\ and $\ddagger$ Department of Mathematics, University of Chicago, Chicago, Illinois 60637 \\ E-mail: lowengrb@math.unc.edu
}

Received February 11, 1999; revised August 3, 1999

We consider the problem of microstructural evolution in binary alloys in two dimensions. The microstructure consists of arbitrarily shaped precipitates embedded in a matrix. Both the precipitates and the matrix are taken to be elastically anisotropic, with different elastic constants. The interfacial energy at the precipitatematrix interfaces is also taken to be anisotropic. This is an extension of the inhomogeneous isotrpic problem considered by H.-J. Jou et al. (1997, J. Comput. Phys. 131, 109). Evolution occurs via diffusion among the precipitates such that the total (elastic plus interfacial) energy decreases; this is accounted for by a modified Gibbs-Thomson boundary condition at the interfaces. The coupled diffusion and elasticity equations are reformulated using boundary integrals. An efficient preconditioner for the elasticity problem is developed based on a small scale analysis of the equations. The solution to the coupled elasticity-diffusion problem is implemented in parallel. Precipitate evolution is tracked by special non-stiff time stepping algorithms that guarantee agreement between physical and numerical equilibria. Results show that small elastic inhomogeneities in cubic systems can have a strong effect on precipitate evolution. For example, in systems where the elastic constants of the precipitates are smaller than those of the matrix, the particles move toward each other, where the rate of approach depends on the degree of inhomogeneity. Anisotropic surface energy can either enhance or reduce this effect, depending on the relative orientations of the anisotropies. Simulations of the evolution of multiple precipitates indicate that the elastic constants and surface energy control precipitate morphology and strongly influence nearest neighbor interactions. However, for the parameter ranges considered, the overall evolution of systems with large numbers of precipitates is primarily driven by the overall reduction in surface energy. Finally,

\footnotetext{
${ }^{1}$ Current address: Department of Mathematics, University of North Carolina, Chapel Hill, NC 275999-3250.

${ }^{2}$ Current address: Department of Mathematics, University of California at Irvine, Irvine, CA 92697-3875.
} 
we consider a problem related to the microstructure of fully orthotropic geological materials. (c) 2000 Academic Press

Key Words: microstructure; anisotropy; inhomogeneity; elasticity, diffusion.

\section{INTRODUCTION}

In this paper, we consider the numerical solution for the problem of microstructural evolution in binary metal alloys that are produced by a solid-state phase transformation. As described in [15], these transformations occur when the temperature of a uniform mixture of materials is lowered into a regime where a two-phase mixture of solids is stable. The system responds by nucleating second phase particles (precipitates) which evolve by the diffusion of matter among the distinct crystal phases until equilibrium is reestablished or diffusion is stopped by further lowering of temperature. Because the resulting two-phase microstructure is a major variable in setting the macroscopic properties of the alloy (i.e., stiffness, strength, and toughness), the transformation process is the basis for the manufacturing of alloys such as steels and superalloys.

The importance of microstructure in setting the properties of the alloy is seen by the following example. In many alloys (especially those used at high temperatures), there is an in situ coarsening process in which a dispersion of very small precipitates evolves to a system consisting of a few very large precipitates in order to decrease the surface energy of the system. This coarsening severely degrades the properties of the alloy and can lead to in service failures.

The details of this coarsening process depend strongly on the elastic properties and crystal structures of the alloy components. Experimental observations of the diffusional evolution of nickel-based superalloy microstructures show cuboidal precipitates aligned in specific crystallographic orientations. This structure arises because of the elastic fields generated by the misfit between the precipitate and matrix crystal lattices and is a reflection of the competition between the elastic and surface energies in lowering the total energy of the system (i.e., the sum of the surface and elastic energies). See, for example, [1, 25, 26, $28,13,6,46]$. There is hope that by carefully choosing the alloy components, it may be possible to use the elastic fields to improve material performance over time through better understanding and control of the transformation process.

In this paper, we investigate the influence of elastic stresses on the diffusional motion of precipitates in the two-phase system in two space dimensions. The precipitate-matrix interfaces are assumed to be sharp and we consider the precipitate and the surrounding matrix phases to be both elastically anisotropic and to have different elastic constants (elastically inhomogeneous). In order to more fully investigate the role of crystalline anisotropy on the transformation process, we also consider anisotropic surface energies at matrix-precipitate interfaces. This is, to our knowledge, the first time both anisotropy and inhomogeneity have been included in a boundary integral (sharp interface) simulation of precipitate motion. One of the features we observe in our simulations is that even small inhomogeneities can strongly influence precipitate evolution.

Most previous work on simulating microstructural evolution in elastic media has focused either on the case of homogeneous elasticity with cubic anisotropy, e.g., [43, 41, 42, 27, $45,44]$ or inhomogeneous, isotropic elasticity, e.g., [15, 20]. In the former, the elastic constants of the two phases are anisotropic, with cubic symmetry, but are identical (elastically 
homogeneous) in the two phases. This case is much easier to treat than the inhomogeneous case because the elastic fields can be calculated by direct calculation of an integral and so it is not necessary to solve any equations to obtain the elastic fields [43, 41, 42]. In the latter case, the elastic constants of the two phases are different but the elasticity is assumed to be isotropic. Even in this isotropic setting, the computation of elastic fields is costly because of the need to solve boundary integral equations over all the precipitate-matrix interfaces. Jou et al. [20] developed an efficient preconditioner for these boundary integral equations and applied a non-stiff time stepping method to successfully track the evolution of microstructures for a relatively large number of particles (approximately 20).

Recently, Schmidt and Gross [37, 38, 39], Orlikowski et al. [36, 31], Li and Chen [23, 24], and Lee $[18,19,17]$ have reported results for elastically anisotropic and inhomogeneous systems. Schmidt and Gross investigated the effect of inhomogeneity on the equilibrium shape and stability of a single precipitate (either in all of space or in a periodic box) in cubic anisotropic media. In their procedure, there is no time evolution as a set of nonlinear equations is obtained and solved (using a quasi-Newton method) for the boundary of a precipitate in equilibrium. Orlikowski et al. investigated the morphological evolution and coarsening statistics of elastically inhimogeneous, cubic anisotropic precipitate-matrix systems in both two and three dimensions. Rather than solving the sharp interface equations, Orlikowski et al. used a phase-field (diffuse interface) approach in which mesoscopic field equations are introduced to mimic the diffusion and elasticity problems. Li and Chen used a similar phase field approach to study morphological evolution and splitting of inhomogeneous precipitates; see also Nishimori and Onuki [30] among others. In a different approach, Lee studied the effect of inhomogeneity and anisotropy on particle morphology and evolution by constructing a "discrete atom method." In this method, atoms are considered as balls connected by elastic springs, and diffusional evolution is simulated by taking a series of discrete Monte-Carlo steps.

In this paper, we generalize the approach used by Jou et al. [15] to study the case of anisotropic, inhomogeneous elasticity. The set-up of the problem is the same as in that paper: the microstructure consists of arbitrarily shaped precipitates growing diffusionally in an elastically stressed matrix. The precipitate-matrix interfaces are assumed to be sharp and coherent. Diffusion of the solute is taken to occur in the matrix only and is assumed to be quasi-static. Elastic stresses may be generated by either far-field applied strains or by mismatch strains between the phases. Unlike [15], the phases are elastically anisotropic with different material constants. The elasticity and composition fields are assumed to interact through an elastic energy term in a generalized Gibbs-Thomson boundary condition for the composition field. This boundary condition includes an anisotropic surface energy following Herring [11]. We refer the reader to [15] for further discussion, implications, and references.

Both the diffusion and elasticity problems for the multi-phase system are reformulated in terms of boundary integral equations; their solutions are used to generate the normal velocity of the interface through a flux-balance condition. As in [15], we use the method of Greenbaum et al. [8] to solve the integral equations for the diffusion problem. However, both the elasticity solver and the non-stiff time stepping algorithms presented here are new, although our approach parallels [15].

To solve the elasticity problem, we use a direct boundary integral representation involving both monopoles and dipoles based on the fundamental solution for orthotropic elasticity given by Green [7] and we use the approach of Rizzo and Shippy [34]. Analogously to the inhomogeneous, isotropic case investigated in [15], four linear integral equations are 
obtained for each interface by using the coherency conditions at the interface, i.e., continuity of both displacements and tractions. Although the structure of the equations is similar to the isotropic inhomogeneous case, the integral kernels in the anisotropic case are more complicated. As is the case with the inhomogeneous isotropic elasticity, these integral equations are not of Fredholm type because they involve Cauchy, logarithmic, and smooth kernels. This leads to the ill-conditioned linear systems. However, following the approach in [15], we perform an analysis of the integral equations at small spatial scales and use the result as a preconditioner to reformulate the integral equations to a second kind Fredholm form. We then use the iterative method GMRES [35] to solve the system.

To track the evolution of the interfaces, we present new non-stiff time integration algorithms. These algorithms are designed to accurately capture certain details of the numerical solutions such as the chemical potential on the interface. We show that these details can sensitively depend on the numerical time integration algorithms. This sensitive dependence was apparently first noticed only recently in [20] in the context of a diffuse interface method. This is likely because macroscopic properties such as particle shapes are much less sensitive to the algorithms.

Finally, we implement our method using parallel computation. The main cost of the algorithm is computing integrals for the diffusion and elasticity integral equations and the normal velocity. If there are $M$ computational points on an interface, then direct summation requires $O\left(M^{2}\right)$ operations to compute each integral at each grid point on the interface. Although fast summation techniques have been developed for isotropic elasticity [9] in which the computational effort is reduced to $O(M)$, there is as yet, no fast algorithm for the anisotropic case due to the form of the integral kernels. To reduce the computational cost, we exploit the easily parallelizable structure of direct summation as follows. Let $P$ be the number of processors. Then, we compute $M / P$ integrals at each processor simultaneously and we broadcast the result to all the other processors. This has a perfect workload balance if $M / P$ is an integer and has a communication load of order $O(M)$. For the $O(M)$ operations, such as updating the interface in time, we perform the computations sequentially. For large numbers of computational nodes, we regularly achieve efficiencies on the order of $90 \%$.

Our results show that small elastic inhomogeneities in cubic systems can have a strong effect on precipitate evolution. For example, in systems where the elastic constants of the precipitates are smaller than those of the matrix, the particles move toward each other, where the rate of approach depends on the degree of inhomogeneity. We find that anisotropic interfacial energy can either enhance or reduce this effect, depending on the relative orientations of the elastic and interfacial anisotropies. However, simulations of the evolution of multiple precipitates indicates that while there are qualitative differences that arise owing to different elastic constants and interfacial anisotropies, the process as a whole is primarily driven by an overall reduction in interfacial energy. Finally, we consider a problem related to the microstructure of fully orthotropic geological materials.

\section{FORMULATION}

In this section, the field equations for the diffusion and elasticity problems and the formulation of the elasticity problem as a boundary integral equation are presented. The diffusion problem and its reformulation as a boundary integral equation are given in [15] and so here we only give a brief review of the relevant equations. The matrix and precipitate 
phases occupy the two-dimensional plane $R^{2}$, with the matrix phase $\Omega^{M}$ extending to infinity and the precipitate phase $\Omega^{P}$ consisting of $p$ separate particles occupying a finite area. The individual interfaces between the two phases are denoted by $\Gamma_{i}, i=1 \ldots p$, with the entire interface given by $\Gamma=\bigcup_{i=1}^{p} \Gamma_{i}$. Each $\Gamma_{i}$ is assumed to be a smooth disjoint closed curve with unit normal vector $n$ taken to point from the precipitate phase to the matrix phase. For the case of precipitate growth, it is convenient to take a circular far boundary $\Gamma_{\infty}$ with radius $R_{\infty}$ and inward normal $\mathbf{n}$ that encloses all the precipitates. This boundary will appear only in the limit $R_{\infty} \rightarrow \infty$ through a far-field mass flux condition for the diffusion field. All variables are dimensionless, with the normalization described in an appendix of [15].

\subsection{Diffusion}

The problem for the diffusion fields is as follows. Let $c$ be a non-dimensional composition. We suppose that the diffusion is quasi-static in the matrix, so

$$
\Delta c=0 \quad \text { in } \Omega^{M}
$$

and we suppose there is no diffusion in the precipitate phase. The boundary condition for the matrix composition at the precipitate-matrix interface is the generalized Gibbs-Thomson condition $[11,22,15,20]$

$$
c=\left(\tau(\theta)+\tau^{\prime \prime}(\theta)\right) \kappa+Z g^{e l} \quad \text { on } \Gamma,
$$

where $\theta$ is the tangent angle to $\Gamma, \tau(\theta)=1+\tau_{0}(\theta)$ is the anisotropic surface energy, $\kappa$ is the mean curvature of the interface, $Z$ characterizes the relative contribution of the elastic and surface energies, and $g^{e l}$ is an elastic energy density defined by Eq. (14) below. The surface energy $\tau(\theta)$ is assumed to be a smooth function of $\theta$ (i.e., no cusps) such that $\tau+\tau^{\prime \prime}>0$.

One may also allow a mass flux $J$ into the system through the far-field condition

$$
-J=\lim _{R_{\infty} \rightarrow \infty} \frac{1}{2 \pi} \int_{\Gamma_{\infty}} \nabla c \cdot \mathbf{n} d s .
$$

Here, $J$ is the total mass flux into the system. If $J>0$ the total area of the precipitates increases with time (growth), while if $J=0$ the total area of the precipitates remains fixed (coarsening). In this paper, we consider only $J=0$.

Finally, the normal velocity $V$ of the precipitate-matrix interface is computed through a flux balance at the interface,

$$
V=\left.\nabla c \cdot \mathbf{n}\right|_{\Gamma}
$$

Equations (1)-(4) can be formulated in terms of boundary integrals by using a dipole density on $\Gamma$ and source terms for the far-field flux. Given $\tau(\theta)$ and $g^{e l}$ from the solution to the elasticity problem, the boundary integral problem for diffusion can be solved numerically, and the normal velocity can be computed by using the Dirichlet-Neumann map. Details are given in $[15,8]$.

\subsection{Elasticity}

In order to calculate the elastic energy density $g^{e l}$ used in Eq. (2), we must first compute the elastic fields on each interface $\Gamma_{j}$. The elastic fields arise because of misfit strains 
between the precipitate and matrix phases as well as far-field applied strains. Misfit is taken into account through the constitutive relations between the stress $\Sigma$ and the strain $\mathcal{E}$. In the precipitate,

$$
\Sigma^{P}=\mathcal{C}^{P}\left(\mathcal{E}^{P}-\mathcal{E}^{T}\right)
$$

where $\mathcal{E}^{T}$ denotes the misfit strain, while in the matrix,

$$
\Sigma^{M}=\mathcal{C}^{M} \mathcal{E}^{M}
$$

In Eqs. (5) and (6), $\mathcal{C}$ denotes the fourth-rank stiffness tensor of the material. The strain components $\mathcal{E}_{i j}^{\chi}$ are computed from the displacement components $u_{i}$ by

$$
\mathcal{E}_{i j}^{\chi}=\frac{1}{2}\left(u_{i, j}^{\chi}+u_{j, i}^{\chi}\right)
$$

with $\chi=P, M$ to denote the the precipitate and matrix, respectively.

In this work, we take both phases to have orthotropic symmetry, i.e., two directions of symmetry. Using compact notation in two dimensions and letting 1 and 2 be the symmetry directions, we write, for $\chi=P, M$,

$$
\Sigma^{\chi}=\left(\begin{array}{c}
\Sigma_{11}^{\chi} \\
\Sigma_{22}^{\chi} \\
\Sigma_{12}^{\chi}
\end{array}\right) \quad \text { and } \quad \mathcal{E}^{\chi}=\left(\begin{array}{c}
\mathcal{E}_{11}^{\chi} \\
\mathcal{E}_{22}^{\chi} \\
\mathcal{E}_{12}^{\chi}
\end{array}\right)
$$

with the stiffness tensor given by

$$
C^{\chi}=\left(\begin{array}{ccc}
c_{11}^{\chi} & c_{12}^{\chi} & 0 \\
c_{12}^{\chi} & c_{22}^{\chi} & 0 \\
0 & 0 & c_{66}^{\chi}
\end{array}\right)
$$

Alternatively, we may define the compliance tensor

$$
S^{\chi}=\left(C^{\chi}\right)^{-1}=\left(\begin{array}{ccc}
s_{11}^{\chi} & s_{12}^{\chi} & 0 \\
s_{12}^{\chi} & s_{22}^{\chi} & 0 \\
0 & 0 & s_{66}^{\chi}
\end{array}\right) .
$$

We remark that if the region $\chi$ has cubic anisotropy then $s_{11}^{\chi}=s_{22}^{\chi}$; for isotropic symmetry $s_{11}^{\chi}=s_{22}^{\chi}$ and $s_{66}^{\chi}=2\left(s_{11}^{\chi}-s_{12}^{\chi}\right)$.

We now give the classical formulation of the elasticity problem in the two-phase domain. In the next subsection, we present the reformulation of the system as boundary integral equations.

The field equations for elasticity are

$$
\nabla \cdot \Sigma^{\chi}=0 \quad \text { in } \Omega^{\chi=M, P}
$$


in the absence of body forces. We assume that the two-phase interface $\Gamma$ is coherent, and so the boundary conditions are given by the continuity of displacement,

$$
\left.\mathbf{u}\right|_{P}=\left.\mathbf{u}\right|_{M},
$$

and traction,

$$
\left.\mathbf{t}\right|_{P}=\Sigma^{P} \mathbf{n}=\Sigma^{M} \mathbf{n}=\left.\mathbf{t}\right|_{M}
$$

Finally we take far-field conditions

$$
\begin{aligned}
& \lim _{r \rightarrow \infty} \mathcal{E}^{M}=\mathcal{E}^{0} \\
& \lim _{r \rightarrow 0} \mathbf{u}^{P}<\infty,
\end{aligned}
$$

where $\mathcal{E}^{0}$ is an applied far-field strain.

Once the elasticity problem is solved, $g^{e l}$ is computed as

$$
g^{e l}=\frac{1}{2} \Sigma^{P}:\left(\mathcal{E}^{P}-\mathcal{E}^{T}\right)-\frac{1}{2} \Sigma^{M}: \mathcal{E}^{M}+\Sigma^{M}:\left(\mathcal{E}^{M}-\mathcal{E}^{P}\right) \quad \text { on } \Gamma \text {. }
$$

Since $g^{e l}$ is only needed on the precipitate-matrix interfaces, it is only necessary to compute the elastic fields on $\Gamma$. This can be done by reformulating Eqs. (11)-(14) onto $\Gamma$ through a system of linear boundary integral equations.

\subsection{Boundary Integral Formulation for Elasticity}

The reformulation of the elasticity problem through boundary integrals stems from the fundamental solution to orthotropic elasticity in two dimensions given by Green and Taylor [7]; see also Rizzo and Shippy [34]. Let $\alpha_{1}$ and $\alpha_{2}$ be positive constants determined from the elastic compliance tensor by

$$
\begin{aligned}
\alpha_{1}+\alpha_{2} & =\left(2 s_{12}+s_{66}\right) / s_{22}, \\
\alpha_{1} \alpha_{2} & =s_{11} / s_{22} .
\end{aligned}
$$

Let $U\left(\mathbf{x}^{\prime}, \mathbf{x}\right)$ be the fundamental solution tensor that generates the displacement vectors $u_{j}$ at $\mathbf{x}^{\prime}$ arising from an isolated force at $\mathbf{x}$, acting in the positive $x_{i}$ direction and with magnitude

$$
\beta=2 \pi\left(\alpha_{1}-\alpha_{2}\right) s_{22}
$$

Then,

$$
u_{j}\left(\mathbf{x}^{\prime}\right)=U_{i j}\left(\mathbf{x}^{\prime}, \mathbf{x}\right) e_{i}(\mathbf{x})
$$

where $e_{i}$ is the base vector along $x_{i}$. This fundamental solution $U$ is given as

$$
\begin{aligned}
& U_{11}=\alpha_{1}^{1 / 2} A_{2}^{2} \log r_{1}-\alpha_{2}^{1 / 2} A_{1}^{2} \log r_{2}, \quad U_{12}=A_{1} A_{2}\left(\theta_{2}-\theta_{1}\right), \\
& U_{21}=A_{1} A_{2}\left(\theta_{2}-\theta_{1}\right), \quad U_{22}=\alpha_{2}^{-1 / 2} A_{2}^{2} \log r_{2}-\alpha_{1}^{-1 / 2} A_{1}^{2} \log r_{1},
\end{aligned}
$$


where

$$
A_{j}=s_{12}-\alpha_{j} s_{22} \quad \text { for } j=1,2
$$

and

$$
r_{j}=\left(x_{1}-x_{1}^{\prime}\right)^{2}+\left(x_{2}-x_{2}^{\prime}\right)^{2} / \alpha_{j}, \quad \theta_{j}=\arctan \left(\left(x_{2}-x_{2}^{\prime}\right) / \sqrt{\alpha_{j}}\left(x_{1}-x_{1}^{\prime}\right)\right) .
$$

Because $\alpha_{1}$ and $\alpha_{2}$ must be positive, Eqs. (15) and (16) may be viewed as restrictions on the elastic constants. For example, let $A$ be a measure of the anisotropy given by

$$
A=2\left(\sqrt{s_{11} s_{22}}-s_{12}\right) / s_{66} .
$$

Then, the restriction on $\alpha_{1}, \alpha_{2} \geq 0$ reduces to requiring $A \leq 1$. Fortunately, for cubic systems, this restriction is not as severe as it appears. In cubic elasticity $s_{11}=s_{22}$ and so rotating the coordinate system by $\pm \pi / 4$ takes $A \rightarrow 1 / A$ while preserving the cubic structure of the stiffness and compliance tensors. This restricts the two-phase problem with cubic anisotropy to cases where the elastic constants of both phases have either $A>1$ or $A<1$. This trick, however, does not work for general orthotropic systems, as rotations create non-orthotropic structure in the stiffness and compliance tensors.

It is straightforward to take derivatives of $U_{i j}$ to find the stresses associated with the displacement $u_{j}$. One may then compute the tractions acting on an arbitrary contour $L$ surrounding the point $\mathbf{x}$ (e.g., see [34]). The traction tensor field $T\left(\mathbf{x}^{\prime}, \mathbf{x}\right)$ is found to be

$$
\begin{aligned}
& T_{11}=\left(x_{k}^{\prime}-x_{k}\right) n_{k}^{\prime}\left(\frac{A_{1}}{\sqrt{\alpha_{2}} r_{2}^{2}}-\frac{A_{2}}{\sqrt{\alpha_{1}} r_{1}^{2}}\right), \quad T_{12}=M_{1} \frac{A_{1}}{\alpha_{1} r_{1}^{2}}-M_{2} \frac{A_{2}}{\alpha_{2} r_{2}^{2}} \\
& T_{21}=M_{1} \frac{A_{2}}{r_{1}^{2}}-M_{2} \frac{A_{1}}{r_{2}^{2}}, \quad T_{22}=\left(x_{k}^{\prime}-x_{k}\right) n_{k}^{\prime}\left(\frac{A_{1}}{\sqrt{\alpha_{1}} r_{1}^{2}}-\frac{A_{2}}{\sqrt{\alpha_{2}} r_{2}^{2}}\right),
\end{aligned}
$$

where $\left(n_{1}^{\prime}, n_{2}^{\prime}\right)$ is the outward unit normal to $L$, and

$$
M_{j}=\alpha_{j}^{1 / 2}\left(x_{1}^{\prime}-x_{1}\right) n_{2}^{\prime}-\alpha_{j}^{-1 / 2}\left(x_{2}^{\prime}-x_{2}\right) n_{1}^{\prime} .
$$

The use of Green's fundamental solution to reformulate the elasticity equations as boundary integrals was derived previously by Rizzo and Shippy [34]. In the absence of misfit and applied strains, the result is as follows. ${ }^{3}$ Let $t_{k}$ be tractions and $u_{k}$ displacements acting on an arbitrary contour $L$. Then for $\mathbf{x}$ inside $L$,

$$
u_{j}(\mathbf{x})=\frac{1}{\beta} \int_{L}\left(t_{k}\left(\mathbf{x}\left(s^{\prime}\right)\right) U_{k j}\left(\mathbf{x}, \mathbf{x}\left(s^{\prime}\right)\right)-u_{k}\left(\mathbf{x}\left(s^{\prime}\right)\right) T_{k j}\left(\mathbf{x}, \mathbf{x}\left(s^{\prime}\right)\right)\right) d s^{\prime},
$$

where we denote the integration point by $\mathbf{x}\left(s^{\prime}\right)$ with $s^{\prime}$ the arclength along $L$. If we let $\mathbf{x} \rightarrow \mathbf{x}(s)$ on $L$

$$
\left.u_{j}(\mathbf{x}(s))=\frac{2}{\beta}\left(\oiint_{L}\left(t_{k}\left(\mathbf{x}\left(s^{\prime}\right)\right) U_{k j}\left(\mathbf{x}(s), \mathbf{x}\left(s^{\prime}\right)\right)-u_{k}\left(\mathbf{x}\left(s^{\prime}\right)\right) T_{k j}\left(\mathbf{x}(s), \mathbf{x}\left(s^{\prime}\right)\right)\right) d s^{\prime}\right)\right)
$$

where the $P$ indicates a principal value integral. It is straightforward to generalize this formula to add misfit and applied strains; both are included in the next subsection.

\footnotetext{
${ }^{3}$ The Einstein summation notation is used: summations are performed over repeated indices.
} 
2.3.1. Application to two-phase problems. Given the fundamental solution for orthotropic elasticity, the formulation of the boundary integral equation for the two-phase problem proceeds as in the isotropic case considered in [15]. Briefly, Eq. (27) is used in both the precipitate and matrix phases with the appropriate elastic constants and applied and misfit strains. Enforcing continuity of displacement and traction results in four coupled integral equations for each interface. This is seen as follows.

Precipitate. Let $\partial \Omega^{P}=\Gamma$ be the boundary of the precipitate $\Omega^{P}$. Applying Eq. (27) to the precipitate boundary and using the constitutive relation Eq. (5), we obtain

$$
\begin{aligned}
& u_{j}^{P}(\mathbf{x}(s))+\frac{2}{\beta^{P}}\left(\oiint_{L} u_{k}^{P}\left(\mathbf{x}\left(s^{\prime}\right)\right) T_{k j}^{P}\left(\mathbf{x}\left(s^{\prime}\right), \mathbf{x}(s)\right) d s^{\prime}-\int_{\Gamma} t_{k}^{P}\left(\mathbf{x}\left(s^{\prime}\right)\right) U_{k j}^{P}\left(\mathbf{x}\left(s^{\prime}\right), \mathbf{x}(s)\right) d s^{\prime}\right) \\
& =\frac{2}{\beta^{P}} \int_{\Gamma} t_{k}^{T}\left(\mathbf{x}\left(s^{\prime}\right)\right) U_{k j}^{P}\left(\mathbf{x}\left(s^{\prime}\right), \mathbf{x}(s)\right) d s^{\prime},
\end{aligned}
$$

where $t_{k}^{P}=\Sigma_{k l}^{P} n_{l}$ is the traction and $t_{k}^{T}=C_{k l m n}^{P} \mathcal{E}_{m n}^{T} n_{l}$ is the misfit traction.

Matrix. The matrix phase requires a little more work. We introduce an artificial far field boundary $\Gamma_{\infty}$ and we decompose the total fields into their uniform far-field component (denoted by a superscript 0) plus a remainder (e.g., see [15] for details). Then for $\mathbf{x}$ in $\Omega^{M}$,

$$
\begin{aligned}
u_{j}^{M}(\mathbf{x})-u_{j}^{0}(\mathbf{x})= & -\frac{1}{\beta^{M}} \int_{\Gamma}\left(\left(t_{k}^{M}\left(\mathbf{x}\left(s^{\prime}\right)\right)-t_{k}^{0}\left(\mathbf{x}\left(s^{\prime}\right)\right)\right) U_{k j}^{M}\left(\mathbf{x}\left(s^{\prime}\right), \mathbf{x}\right)-\left(u_{k}^{M}\left(\mathbf{x}\left(s^{\prime}\right)\right)\right.\right. \\
& \left.\left.-u_{k}^{0}\left(\mathbf{x}\left(s^{\prime}\right)\right)\right) T_{k j}^{M}\left(\mathbf{x}\left(s^{\prime}\right), \mathbf{x}\right)\right) d s^{\prime}+\frac{1}{\beta^{M}} \int_{\Gamma_{\infty}}\left(\left(t_{k}^{M}\left(\mathbf{x}\left(s^{\prime}\right)\right)-t_{k}^{0}\left(\mathbf{x}\left(s^{\prime}\right)\right)\right)\right. \\
& \left.\times U_{k j}^{M}\left(\mathbf{x}\left(s^{\prime}\right), \mathbf{x}\right)-\left(u_{k}^{M}\left(\mathbf{x}\left(s^{\prime}\right)\right)-u_{k}^{0}\left(\mathbf{x}\left(s^{\prime}\right)\right)\right) T_{k j}^{M}\left(\mathbf{x}\left(s^{\prime}\right), \mathbf{x}\right)\right) d s^{\prime},
\end{aligned}
$$

where $\mathbf{u}_{k}^{0}=\mathcal{E}_{k l}^{0} x_{l}$ and analogously for $t_{k}^{0}$. We assume that the applied strain $\mathcal{E}^{0}$ is constant.

It can be shown that as $\Gamma_{\infty}$ goes to $\infty$, the integral over $\Gamma_{\infty}$ tends to a constant independent of $\mathbf{x}$ [2]. This implies that the integral over $\Gamma_{\infty}$ contributes a constant to the displacement, and so can be neglected. Then, letting $\mathbf{x}$ approach $\Gamma$ from $\Omega^{M}$, we have

$$
\begin{aligned}
u_{j}^{M}(\mathbf{x}(s))-\frac{2}{\beta^{M}}\left(\not \oiint_{\Gamma} u_{k}^{M}\left(z^{\prime}\right) T_{k j}^{M}\left(\mathbf{x}\left(s^{\prime}\right), \mathbf{x}(s)\right) d s^{\prime}-\int_{\Gamma} t_{k}^{M}\left(\mathbf{x}\left(s^{\prime}\right)\right) U_{k j}^{M}\left(\mathbf{x}\left(s^{\prime}\right), \mathbf{x}(s)\right) d s^{\prime}\right) \\
=u_{j}^{0}(\mathbf{x}(s))-\frac{2}{\beta^{M}}\left(\oint_{\Gamma} u_{k}^{0}\left(\mathbf{x}\left(s^{\prime}\right)\right) T_{k j}^{M}\left(\mathbf{x}\left(s^{\prime}\right), \mathbf{x}(s)\right) d s^{\prime}\right. \\
\left.\quad-\int_{\Gamma} t_{k}^{0}\left(\mathbf{x}\left(s^{\prime}\right)\right) U_{k j}^{M}\left(\mathbf{x}\left(s^{\prime}\right), \mathbf{x}(s)\right) d s^{\prime}\right) .
\end{aligned}
$$

Coherency conditions. Since $u_{k}^{P}=u_{k}^{M}$ and $t_{k}^{P}=t_{k}^{M}$ on $\Gamma$, Eqs. (28) and (30) yield a system of four linear integral equations for the single-valued displacement and traction fields on $\Gamma$. 
Existence and uniqueness of solutions. Although this system is not Fredholm since $T^{P}$ and $T^{M}$ contain a term with a Cauchy singularity, it can be transformed to a secondkind Fredholm equation using the small scale decomposition given in the next subsection. Classical Fredholm theory [33] can then be used to show that solutions exist and are unique ( $\mathbf{u}^{0}$ is specified at infinity, ensuring uniqueness).

\subsection{Small Scale Preconditioner for the Elasticity Integral Equations}

We can analyze the system (28)-(30) at small spatial scales in an analogous way to the isotropic system presented in [15]. The idea is to determine and separate the dominant terms at small spatial scales. Roughly speaking, these dominant terms are those terms associated with singular integral kernels. For example, integral operators with nonsingular kernels are smoothing at high Fourier wavenumbers (small spatial scales) while those with singular kernels smooth much less (if at all). Thus, at small scales, operators with singular kernels are dominant. We refer the reader to [15] for a full discussion of this procedure.

Let the boundary displacements $\mathbf{u}=\mathbf{u}^{P}=\mathbf{u}^{m}$ and tractions $\mathbf{t}=\mathbf{t}^{P}=\mathbf{t}^{M}$. Then, Eqs. (28) and (30) can be written as

$$
\mathcal{K}(\mathbf{u}, \mathbf{t})=\mathbf{f}
$$

with

$$
\begin{gathered}
\mathcal{K}(\mathbf{u}, \mathbf{t})=\left(\begin{array}{c}
u_{j}+\mathcal{K}_{j}^{P}\left(u_{k}, t_{k}\right) \\
u_{j}-\mathcal{K}_{j}^{M}\left(u_{k}, t_{k}\right)
\end{array}\right), \quad \mathbf{f}=\left(\begin{array}{c}
f_{j}^{P} \\
f_{j}^{M}
\end{array}\right), \\
\mathcal{K}_{j}^{\mathcal{X}}\left(u_{k}, t_{k}\right)=\frac{2}{\beta^{\mathcal{X}}}\left(\oiint_{\Gamma} u_{k}\left(z^{\prime}\right) T_{k j}^{\mathcal{X}}\left(z^{\prime}, z\right) d s-\int_{\Gamma} t_{k}\left(z^{\prime}\right) U_{k j}^{\mathcal{X}}\left(z^{\prime}, z\right) d s\right), \\
f_{j}^{P}=\mathcal{K}_{j}^{P}\left(0, t_{k}^{T}\right), \quad f_{j}^{M}=u_{j}^{0}-\mathcal{K}_{j}^{M}\left(u_{k}^{0}, t_{k}^{0}\right) .
\end{gathered}
$$

We now analyze the smoothness of the kernels. We suppose that the interfaces are well separated and do not self-intersect so that $z(s) \neq z\left(s^{\prime}\right)$ unless $s=s^{\prime}$.

We first notice that $U_{12}\left(z(s), z\left(s^{\prime}\right)\right), U_{21}\left(z(s), z\left(s^{\prime}\right)\right), T_{11}\left(z(s), z\left(s^{\prime}\right)\right)$, and $T_{22}\left(z(s), z\left(s^{\prime}\right)\right)$ contain no singularities and remain smooth as $s \rightarrow s^{\prime}$. The remaining kernels $U_{11}, U_{22}, T_{12}$, and $T_{21}$ do become singular as $s \rightarrow s^{\prime}$. Their structure is obtained as follows.

Let $0 \leq \alpha \leq 2 \pi$ parameterize $\Gamma$, i.e., $s=s(\alpha)$. Then, observe that

$$
r_{j}^{2} \sim\left(x_{1 \alpha}^{2}+x_{2 \alpha}^{2} / \alpha_{j}\right)\left(\alpha-\alpha^{\prime}\right)^{2} \quad \text { as } \alpha \rightarrow \alpha^{\prime},
$$

where the $\alpha_{j}$ are determined from elastic constants via Eqs. (15)-(16) and are not related to the parameter $\alpha$. Also, we use the notation $\sim$ to denote that the difference between two is a smoothing operator (integral operator with a smooth kernel) [15]. By using Eq. (35), one can show that

$$
\begin{aligned}
& T_{12}=\left(\frac{A_{1}}{\sqrt{\alpha_{1}}}-\frac{A_{2}}{\sqrt{\alpha_{2}}}\right) \frac{1}{2 s_{\alpha}} \cot \left(\frac{\alpha-\alpha^{\prime}}{2}\right)+\text { smooth function, } \\
& T_{21}=\left(A_{2} \sqrt{\alpha_{1}}-A_{1} \sqrt{\alpha_{2}}\right) \frac{1}{2 s_{\alpha}} \cot \left(\frac{\alpha-\alpha^{\prime}}{2}\right)+\text { smooth function, }
\end{aligned}
$$


Now, define the periodic Hilbert transform by

$$
\mathcal{H}(\mu) \equiv \frac{1}{2 \pi} \int_{0}^{2 \pi} \mu(\alpha) \cot \frac{1}{2}\left(\alpha-\alpha^{\prime}\right) d \alpha^{\prime} .
$$

Then, we obtain

$$
\begin{aligned}
& \int_{\Gamma} \mu T_{12} d s \sim\left(\frac{A_{1}}{\sqrt{\alpha_{1}}}-\frac{A_{2}}{\sqrt{\alpha_{2}}}\right) \pi \mathcal{H}(\mu), \\
& \int_{\Gamma} \mu T_{21} d s \sim\left(\sqrt{\alpha_{1}} A_{2}-\sqrt{\alpha_{2}} A_{1}\right) \pi \mathcal{H}(\mu) .
\end{aligned}
$$

This is the small scale decomposition of the integral operators with kernels $T_{12}$ and $T_{21}$.

It should be observed that the Hilbert transform is not smoothing at small scales. Let $\hat{\mu}(k)$ denote the Fourier transform of $\mu$ at wavenumber $k$. Then,

$$
\hat{\mathcal{H}}(\mu)(k)=-i \operatorname{sgn}(k) \hat{\mu}(k)
$$

and so high frequencies are not "damped" as they would be for a smoothing operator. For a smoothing operator $S(\mu)$, we have $\hat{S}(\mu)(k)=O\left(e^{-\rho|k|} \sum_{l} \hat{\mu}(l) e^{-\rho|l|}\right)$ where $\rho$ denotes the strip width of analyticity of the kernels (in the Im $\alpha$-plane) [15]. This justifies the decomposition.

The small scale decompositions for the operators with kernels $U_{11}$ and $U_{22}$ are obtained by performing integration by parts on the terms $\log \left(r_{j}\right)$ and arguing similarly. We obtain

$$
\int_{\Gamma} \mu U_{11} d s \sim\left(\alpha_{1}^{1 / 2} A_{2}^{2}-\alpha_{2}^{1 / 2} A_{1}^{2}\right) \pi s_{\alpha} \mathcal{H}(\sigma)
$$

and

$$
\int_{\Gamma} \mu U_{22} d s \sim\left(\alpha_{2}^{-1 / 2} A_{2}^{2}-\alpha_{1}^{-1 / 2} A_{1}^{2}\right) \pi s_{\alpha} \mathcal{H}(\sigma),
$$

where $\sigma(\alpha)=\int_{0}^{\alpha} \mu\left(\alpha^{\prime}\right) d \alpha^{\prime}$ and we have assumed that $\int_{0}^{2 \pi} \mu\left(\alpha^{\prime}\right) d \alpha^{\prime}=0$. This assumption is valid because in Eqs. (28)-(30), these operators are applied to the tractions $t_{i}$ which have zero mean.

Putting everything together, we conclude

$$
\mathcal{K}_{j}^{\mathcal{X}}\left(u_{k}, t_{k}\right) \sim \mathcal{L}_{j}^{\mathcal{X}}\left(u_{k}, t_{k}\right) \equiv D_{k j}^{\mathcal{X}} \bar{\delta}_{k j 3} \mathcal{H}\left(u_{k}\right)-\delta_{k j} E_{k j}^{\mathcal{X}} s_{\alpha} \mathcal{H}\left(\sigma_{k}\right),
$$

where $\sigma_{k}(\alpha)=\int_{0}^{\alpha} t_{k}\left(\alpha^{\prime}\right) d \alpha^{\prime}$. Further,

$$
\delta_{l k}=\left\{\begin{array}{ll}
1, & l=k \\
0, & l \neq k
\end{array} \quad \bar{\delta}_{l k 3}= \begin{cases}1, & l=1, k=2 \\
-1, & l=2, k=1 \\
0, & l=k\end{cases}\right.
$$

and $D_{i j}^{\mathcal{X}}, E_{i j}^{\mathcal{X}}$, with $\mathcal{X}=P, M$, have nonzero components given by

$$
\begin{array}{ll}
D_{12}=\frac{2 \pi}{\beta}\left(\frac{A_{1}}{\sqrt{\alpha_{1}}}-\frac{A_{2}}{\sqrt{\alpha_{2}}}\right), & D_{21}=\frac{2 \pi}{\beta}\left(\sqrt{\alpha_{2}} A_{1}-\sqrt{\alpha_{1}} A_{2}\right), \\
E_{11}=\frac{2 \pi}{\beta}\left(\alpha_{1}^{1 / 2} A_{2}^{2}-\alpha_{2}^{1 / 2} A_{1}^{2}\right), & E_{22}=\frac{2 \pi}{\beta}\left(\alpha_{2}^{-1 / 2} A_{2}^{2}-\alpha_{1}^{-1 / 2} A_{1}^{2}\right),
\end{array}
$$


where we drop the superscript $\chi$ but note that $\alpha_{i}, A_{i}$, and $\beta$ all depend on the elastic constants.

Recalling Eq. (32), we now write the small scale decomposition of the full elasticity system as

$$
\mathcal{K}(\mathbf{u}, \mathbf{t})=\mathcal{L}(\mathbf{u}, \mathbf{t})+\mathcal{R}
$$

where

$$
\mathcal{L}(\mathbf{u}, \mathbf{t})=\left(\begin{array}{l}
u_{j}+\mathcal{L}_{j}^{P}\left(u_{k}, t_{k}\right) \\
u_{j}-\mathcal{L}_{j}^{M}\left(u_{k}, t_{k}\right)
\end{array}\right)
$$

dominates the small scales and $\mathcal{R}$ is a smoothing operator.

The primary advantage of this formulation is that for a wide range of elastic parameters, $\mathcal{L}$ can be easily inverted in Fourier space when $s_{\alpha}$ is constant in $\alpha$ (as in the equal arclength frame discussed in the next section) by using identities like (41). In this frame, computing $\mathcal{L}^{-1}$ reduces to inverting a $4 \times 4$ matrix at each wavenumber $k$ for each interface. See the Appendix for this matrix.

Using the small scale decomposition, the elasticity integral equations can be reformulated as

$$
\mathcal{L}^{-1} \mathcal{K}(\mathbf{u}, \mathbf{t})=\left[\mathbf{I}+\mathcal{L}^{-1} \mathcal{R}\right](\mathbf{u}, \mathbf{t})=\mathcal{L}^{-1} \mathbf{f}
$$

where $\mathcal{L}^{-1} \mathcal{R}$ is also a smoothing operator [15]. This is a Fredholm integral equation of the 2nd kind.

Computationally, we use $\mathcal{L}^{-1}$ as a preconditioner for the elasticity system. This is efficient because applying $\mathcal{L}^{-1}$ on each interface requires $O(M \log (M))$ operations where $M$ is the total number of grid points on the interface(s) $\Gamma$. This is to be compared with computing $\mathcal{K}$ which requires $O\left(M^{2}\right)$ operations.

\subsection{Energy}

Finally, we assert that the system (1)-(4) and (14) evolves to reduce the sum of surface and elastic energies

$$
W_{t o t}=\int_{\Gamma} \tau(\theta) d s+Z \sum_{\chi=M, P} \int_{\Omega \chi} \Sigma_{i j}^{\chi} \tilde{\mathcal{E}}_{i j}^{\chi} d A,
$$

where

$$
\tilde{\mathcal{E}}^{\chi}= \begin{cases}\mathcal{E}^{P}-\mathcal{E}^{T} & \text { for } \chi=P, \\ \mathcal{E}^{M} & \text { otherwise. }\end{cases}
$$

Following [15], the elastic energy can be rewritten in terms of a boundary integral. We do not give the details here. Lastly, a straightforward calculation shows that

$$
\begin{aligned}
\dot{W}_{t o t} & =\frac{d}{d t} W_{t o t}=\int_{\Gamma}\left(\tau \kappa+Z g^{e l}\right) V d s \\
& =-\int_{\Omega^{M}}|\nabla c|^{2} d A
\end{aligned}
$$

which justifies our assertion above. 


\section{NUMERICAL METHODS}

In this section, we describe the numerical methods used to solve the moving boundary problem. The numerical procedure is the following. (1) The elasticity integral equations are solved to generate $g^{e l}$. (2) The composition integral equations are solved to obtain $V$, the normal velocity of the precipitate/matrix interfaces. (3) The interfaces are updated and the process is repeated. Because the first two procedures rely on well-established techniques we discuss them very briefly and we focus primarily on the time-stepping algorithms for the interface-update and on parallelization issues.

\subsection{Integral Equations}

The integral equations for both elasticity and diffusion are solved using the collocation method together with the iterative solver GMRES [35]. In the elasticity case, the preconditioner $\mathcal{L}$ is used in Fourier space. In the diffusion case, the preconditioner developed by Greenbaum et al. [8] is used. The implementation of these two methods together is completely analogous to that in [15] and the normal velocity $V$ is calculated in the same way as in that paper. We refer the reader to that paper for additional background. The integrals (except in the preconditioners) are calculated by alternating point quadratures [40]. For the integrals involving $\log r_{j}$ kernels we first perform integration by parts in order to reduce the kernels to $1 / r_{j}$-type behavior [15]. This yields spectral accuracy. The reason for using the alternating point quadrature is that it avoids evaluating the integrands at the point of removable singularity and we find it gives slightly better numerical performance. Any derivatives or anti-derivatives are obtained using the FFT. Finally, following [15, 12], a 25th order Fourier filter is employed to reduce aliasing errors.

\section{2. $\theta-A$ Formulation and Small Scale Decomposition of Evolution Equations}

To evolve the precipitate/matrix interfaces $\Gamma$, we follow $[15,12]$ and use the $\theta-A$ formulation in the scaled arclength frame. Here, $\theta$ is the tangent angle to the interface (measured counterclockwise from the horizontal) and $A$ is the area of a precipitate. To briefly review, let $\alpha$ parametrize the interface. Then,

$$
\theta(\alpha, t)=\tan ^{-1}\left(x_{2, \alpha} / x_{1, \alpha}\right) \quad \text { and } \quad A(t)=\frac{1}{2} \int_{\Gamma}\left(x_{1}, x_{2}\right) \cdot \mathbf{n} d s
$$

We evolve the interface $\Gamma$ in the scaled arclength frame by choosing the tangential velocity $T$ to be

$$
T(\alpha, t)=\frac{\alpha}{2 \pi} \int_{0}^{2 \pi} \theta_{\alpha^{\prime}} V d \alpha^{\prime}-\int_{0}^{\alpha} \theta_{\alpha} V d \alpha^{\prime}
$$

This maintains the relation

$$
s_{\alpha}(\alpha, t)=\frac{L(t)}{2 \pi},
$$

where $L(t)$ is the length of the interface. This keeps computational points on the curve equally spaced in arclength at all times. 
The length $L(t)$ is found from $\theta$ and $A(t)$ by using the relation

$$
\begin{aligned}
A(t)= & \frac{L^{2}(t)}{8 \pi^{2}} \int_{0}^{2 \pi}\left[\sin (\theta(\alpha, t)) \int_{0}^{\alpha^{\prime}} \cos \left(\theta\left(\alpha^{\prime}, t\right)\right) d \alpha^{\prime}\right. \\
& \left.-\cos (\theta(\alpha, t)) \int_{0}^{\alpha^{\prime}} \sin \left(\theta\left(\alpha^{\prime}, t\right)\right) d \alpha^{\prime}\right] d \alpha .
\end{aligned}
$$

The interface coordinates $\left(x_{1}, x_{2}\right)$ are reconstructed from $\theta$ and $A$ by integrating in $\alpha$ the expressions

$$
x_{1, \alpha}=\frac{L}{2 \pi} \cos (\theta(\alpha, t)) \quad \text { and } \quad x_{2, \alpha}=\frac{L}{2 \pi} \sin (\theta(\alpha, t)),
$$

where the constant of integration is obtained by evolving the centroid $\left(\bar{x}_{1}, \bar{x}_{2}\right)$ separately. The evolution is then given by

$$
\begin{aligned}
\dot{\theta}(\alpha, t) & =\frac{2 \pi}{L}\left(V_{\alpha}+T \theta_{\alpha}\right), \\
\dot{A}(t) & =\int_{\Gamma} V d s, \\
\dot{\bar{x}}_{1} & =\frac{1}{A(t)}\left[\int_{\Gamma} x_{1} V d s-\bar{x}_{1} \dot{A}\right] \\
\dot{\bar{x}}_{2} & =\frac{1}{A(t)}\left[\int_{\Gamma} x_{2} V d s-\bar{x}_{2} \dot{A}\right],
\end{aligned}
$$

where $d s=(L(t) / 2 \pi) d \alpha$.

In [12], it was shown that at small scales

$$
V \sim\left(\frac{2 \pi}{L}\right)^{2} \mathcal{H}\left[\left(\tau+\tau^{\prime \prime}\right) \theta_{, \alpha \alpha}\right]
$$

and thus the evolution Eq. (58) for $\theta$ is stiff. That is, for an explicit time stepping algorithm, the constraint $\Delta t \leq(h L(t) / 2 \pi)^{3}$, where $\Delta t$ and $h$ are the temporal and spatial grid sizes, respectively, must be imposed to maintain stability. An advantage of using the scaled arclength formulation is that it is straightforward to develop non-stiff time integration schemes for Eq. (58). For example, in [12, 15], a time stepping method based on an integrating factor approach was used to remove the stiffness. We note that the remaining equations are not stiff and any explicit time integration scheme can be used to solve them.

\subsection{Time Discretizations}

Taking advantage of the small scale decomposition (62) and the fact that $\tau+\tau^{\prime \prime}=$ $1+\left(\tau_{0}+\tau_{0}^{\prime \prime}\right)$, one can rewrite Eq. $(58)$ as

$$
\begin{aligned}
\theta_{t} & =\left(\frac{2 \pi}{L_{i}}\right)^{3} \mathcal{H}\left[\theta_{\alpha \alpha \alpha}\right]+N(\alpha, t), \quad \text { where } \\
N(\alpha, t) & =\frac{2 \pi}{L}\left(V_{\alpha}+T \theta_{\alpha}\right)-\left(\frac{2 \pi}{L}\right)^{3} \mathcal{H}\left[\theta_{\alpha \alpha \alpha}\right] .
\end{aligned}
$$


Except for the anisotropic surface energy contribution, the remainder terms $N$ are "lower order" at small spatial scales. Although the anisotropic surface energy term $\mathcal{H}\left[\left(\tau_{0}+\right.\right.$ $\left.\left.\tau_{0}^{\prime \prime}\right) \theta_{, \alpha \alpha}\right]_{, \alpha}$ is of the same order as the term $\mathcal{H}\left[\theta_{, \alpha \alpha \alpha}\right]$, we find the splitting in Eq. (63) to be effective numerically provided $\tau_{0}+\tau_{0}^{\prime \prime}$ is smooth and not too close to 1 .

Note that the term $\mathcal{H}\left[\theta_{, \alpha \alpha \alpha}\right]$ is diagonalized by the Fourier transform and so in Fourier space, we obtain

$$
\hat{\theta}_{t}=-m I^{\prime}(t) \hat{\theta}+\hat{N}(\theta)
$$

where $m=(2 \pi k)^{3}$ and

$$
I(t)=\int_{0}^{t} d t^{\prime} / L^{3}\left(t^{\prime}\right)
$$

Thus, in Fourier space, implicit time discretizations can be easily applied. However, some care must be taken with these temporal discretizations so that certain properties of the continuous equations are maintained on the discrete level. For example, in equilibrium, there is an exact balance between the linear and nonlinear terms

$$
\frac{m}{\left(L^{*}\right)^{3}} \hat{\theta}^{*}=\hat{N}\left(\theta^{*}\right)
$$

where the $*$ denotes the equilibrium value. In fact, this balance characterizes the equilibrium solution. Since we are interested in equilibrium microstructures, this balance should also hold on the discrete level. It turns out that this requires some care. For example, in the context of a diffuse interface model, it was observed in [20] that the integrating factor method used in $[12,15]$ violates an analogous version of this balance. This also occurs in the boundary integral context. We refer to this method as IF:

Method IF,

$$
\hat{\tilde{\theta}}^{n+1}=e^{-m\left(I^{n+1}-I^{n}\right)} \hat{\tilde{\theta}}^{n}+\frac{\Delta t}{2}\left[3 e^{-m\left(I^{n+1}-I^{n}\right)} \hat{N}^{n}-e^{-m\left(I^{n+1}-I^{n-1}\right)} \hat{N}^{n-1}\right]
$$

which was originally given in [12]. This method is 2nd order accurate in time [12]. It is straightforward to see that the IF method violates Eq. (67) in equilibrium. Using the same $*$ notation, we find the following discrete balance for the IF method in equilibrium

$$
\left[1-e^{-m \Delta t /\left(L^{*}\right)^{3}}\right] \hat{\tilde{\theta}}^{*}=\frac{\Delta t}{2}\left[3 e^{-m \Delta t /\left(L^{*}\right)^{3}}-e^{-2 m \Delta t /\left(L^{*}\right)^{3}}\right] \hat{N}\left[\tilde{\theta}^{*}\right] .
$$

Equation (69) is a 2nd order approximation to Eq. (67). In fact,

$$
\frac{m}{\left(L^{*}\right)^{3}} \hat{\tilde{\theta}}^{*}-\hat{N}\left[\tilde{\theta}^{*}\right]=\left[\frac{m}{\left(L^{*}\right)^{3}}-\frac{2}{\Delta t} \frac{\left(e^{m \Delta t /\left(L^{*}\right)^{3}}-1\right)}{\left(3-e^{\left.-m \Delta t /\left(L^{*}\right)^{3}\right)}\right.}\right] \hat{\tilde{\theta}}^{*} .
$$

For large $m$, the dominant error term comes from the term $\frac{2}{3 \Delta t}\left[e^{m \Delta t /\left(L^{*}\right)^{3}}-1\right] \hat{\tilde{\theta}}^{*}$. Thus, the error will be small if either $\hat{\tilde{\theta}}^{*}$ is exponentially small (large $m$ ) or $m \Delta t$ is small. Recall that $m=\left(2 \pi|k|^{3}\right)$ where $k$ is the Fourier wavenumber. If large gradients are present in $\tilde{\theta}$, such as would be associated with formation of "corners" (regions of high curvature) in the precipitate-matrix interfaces, the exponential decay of $\hat{\tilde{\theta}}^{*}(k)$ may only be achieved for 
rather large values of the wavenumber $k$ (and hence $m$ ). For example, suppose exponential decay is observed for $|k|>k^{*}$. Then, the right hand side of Eq. (70) (i.e., the error term) is small only if $m\left(k^{*}\right) \Delta t \ll 1$. This is an accuracy condition. If this condition is violated, then the error term can be significant. Typically, the composition on the interface is sensitive to this error term while the interface position and curvature are not. This will be shown in the next section.

If $k^{*}$ is large, then the accuracy condition $m\left(k^{*}\right) \Delta t \ll 1$ can be very restrictive. As indicated above, this restriction is due to the fact that the IF method overdamps the numerical solution. By reducing the amount of damping, this accuracy restriction can be removed completely while the overall order of accuracy is maintained. Of course, reducing the damping narrows the stability region of the numerical scheme. Consider the following Crank-Nicholson type scheme:

Method CN,

$$
\hat{\tilde{\theta}}^{n+1}=\frac{1-\Delta t m /\left(L^{n-1}\right)^{3}}{1+\Delta t m /\left(L^{n+1}\right)^{3}} \hat{\tilde{\theta}}^{n-1}+\frac{2 \Delta t}{1+\Delta t m /\left(L^{n+1}\right)^{3}} \hat{N}^{n} .
$$

Like the IF scheme, the $\mathrm{CN}$ scheme is 2 nd order accurate in time. However, at the highest modes, there is little damping of the linear term and only algebraic damping of the nonlinear term. We find that because there is less damping, smaller time steps (than those used with the IF method) are required for stability. This is discussed further in the next section. It is straightforward to see that numerical equilibria of the $\mathrm{CN}$ scheme satisfy the equilibrium condition (67) exactly.

In a sense, the IF and $\mathrm{CN}$ schemes represent extremes in the amount of damping of the linear and nonlinear terms for 2 nd order methods. One can also derive 2 nd order schemes in which the amount of damping varies between these two extremes yet the numerical equilibrium still satisfies the condition (67) exactly. For example, suppose one wishes to have exponential damping on the nonlinear term. Then, one can consider the scheme

$$
\hat{\tilde{\theta}}^{n+1}=L_{m, n} \hat{\widetilde{\theta}}^{n-1}+2 \Delta t e^{-m\left(I^{n+1}-I^{n}\right)} \hat{N}^{n}
$$

which is analogous to the $\mathrm{CN}$ scheme applied to Eq. (65) using an integrating factor. However, the Fourier coefficient $L_{m, n}$ is chosen so that Eq. (67) is satisfied exactly for the numerical solution. It is straightforward to see that

$$
L_{m, n}=\left[1-m\left(I^{n+1}-I^{n-1}\right) e^{-m\left(I^{n+1}-I^{n-1}\right) / 2}\right]
$$

is the appropriate choice. This leads to the scheme

Method IF2,

$$
\hat{\tilde{\theta}}^{n+1}=\left[1-m\left(I^{n+1}-I^{n-1}\right) e^{-m\left(I^{n+1}-I^{n-1}\right) / 2}\right] \hat{\tilde{\theta}}^{n-1}+2 \Delta t e^{-m\left(I^{n+1}-I^{n}\right)} \hat{N}^{n}
$$

which is 2nd order accurate. Like the $\mathrm{CN}$ scheme, there is little damping on the linear term at the highest wavenumbers. However, like the IF method (and unlike the CN scheme), the nonlinear terms are damped exponentially. As we show in the next section, this scheme has a larger stability region than the $\mathrm{CN}$ method, although it still requires a smaller time step for stability than the IF method. 
The method we find to perform the best, in that the time step required for stability is essentially that of the IF method and that the numerical equilibria satisfy Eq. (67) exactly, is given as follows. We take exponential damping on the linear term and algebraic damping for the nonlinear term. One way to do this which maintains 2 nd order accuracy is to use the scheme:

$$
\begin{aligned}
& \text { Method IF3, } \\
& \hat{\tilde{\theta}}^{n+1}=\frac{1}{1+m\left(I^{n+1}-I^{n-1}\right) e^{m\left(I^{n}-I^{n-1}\right)}} \hat{\tilde{\theta}}^{n-1}+2 \Delta t \frac{\hat{N}^{n}}{m\left(I^{n+1}-I^{n-1}\right)+e^{-m\left(I^{n}-I^{n-1}\right)}} .
\end{aligned}
$$

This is the time-stepping method that we will use primarily in our nonlinear simulations.

\subsection{Parallel Implementation}

We now discuss the issues involved in implementing our method efficiently in parallel. Recall that at each time step, we (1) solve the integral equations for elasticity to obtain $g^{e l}$ on $\Gamma$; (2) solve the integral equations for diffusion to find the composition on $\Gamma$; (3) calculate the normal velocity of $\Gamma$; and (4) update $\Gamma$. The dominant computational cost is associated with steps (1)-(3). In particular, if $M$ is the total number of grid points on $\Gamma$, then the operation count for each of steps (1)-(3) is $O\left(M^{2}\right)$ while the operation count for step (4) is $O(M)$. We note that solving the integral equations for elasticity has the highest computational cost because there are $4 M$ unknowns. Consequently, we implement (1)-(3) in parallel and (4) sequentially.

As an example, consider the integral equations for elasticity. Using a collocation method with an iteration procedure to solve integral equations, a sum that must be performed at each iteration step is

$$
\sum_{i=1}^{M} t_{2}\left(\mathbf{x}\left(s_{i}\right)\right) U_{21}^{\chi}\left(\mathbf{x}\left(s_{i}\right), \mathbf{x}\left(s_{l}\right)\right)
$$

for $l=1, \ldots, M$. Thus, for each $l$, a total of $M$ summations must be performed with each summation taking $O(M)$ work. We use the following strategy for the parallel implementation of the $M$ summations. First, we distribute all $t_{2}$ as well as all other necessary quantities, e.g., the location of the all interfaces $\mathbf{x}(s)$, to every processor. Second, we compute $M / P$ integrals at each processor simultaneously, where $P$ denotes the total number of processors. Third, after the iteration procedure we broadcast the new $t_{k}$ from one processor to all other processors for the next iteration. This strategy has a perfect work-load balance if $M / P$ is an integer and has a communication load of order $O(M)$. We apply the same strategy to all the other summations required to solve the integral equations for elasticity, diffusion and to compute the normal velocity. This strategy has been successfully employed for other problems involving computation of boundary integrals [29].

\section{PERFORMANCE AND CONVERGENCE STUDIES}

In this section, we consider the impact of preconditioning, time stepping, and parallelization on the numerical results, and we verify the accuracy and convergence of the elasticity solver.

Unless otherwise stated, all numerical tests in this section are performed using a dilatational misfit strain $\epsilon_{11}^{T}=\epsilon_{22}^{T}=1, \epsilon_{12}^{T}=0$ with no applied fields and no far field flux $(J=0)$. 
The elastic constants are chosen to have cubic symmetry in both the matrix and precipitate phase. We use $c_{11}^{M}=c_{22}^{M}=1.98, c_{12}^{M}=1.18, c_{66}^{M}=1$, and $c_{11}^{P}=c_{22}^{P}=3.03, c_{12}^{P}=1.61$, and $c_{66}^{P}=1.35$. These elastic constants (scaled by $c_{66}^{M}$ ) are appropriate for a Nickel matrix and $\mathrm{Ni}_{3} \mathrm{Si}$ precipitates $[43,32]$. All precipitates are taken to have the same elastic constants. The surface tension given in Eq. (2) is chosen to allow four-fold symmetry

$$
\tau(\theta)=1+\tau_{0} \cos 4\left(\theta-\theta_{0}\right) .
$$

The values of $\tau_{0}$ and $\theta_{0}$, as well as $Z$, are chosen for each numerical experiment. The precipitates are initially circular with radius of unity. The GMRES error tolerance is $10^{-6}$ and we use 25th order Fourier smoothing with the filter level $10^{-11}$ [12].

\subsection{The Effect of Preconditioning}

Figure 1 shows the iteration count for solving the elasticity equations using GMRES versus time, both with preconditioning and without preconditioning. For this study, $\tau_{0}=0.05$ and $\theta_{0}=0$ for the surface energy, and we consider a single precipitate system with $Z=10$. The temporal scheme used is method IF with the number of grid points $N=512$ and the time step $\Delta t=10^{-4}$. The tolerance level for GMRES is $10^{-10}$. The precipitate starts from a circle and evolves toward a square shape, as expected from the four-fold symmetry of the problem [3]. At the final time $t=0.05$, the precipitate reaches its steady state. Without preconditioning, the iteration count starts at around 45 and decreases to about 6 at $t=0.05$. With preconditioning, the initial iteration count is 11 (a factor of 4 improvement) and decreases

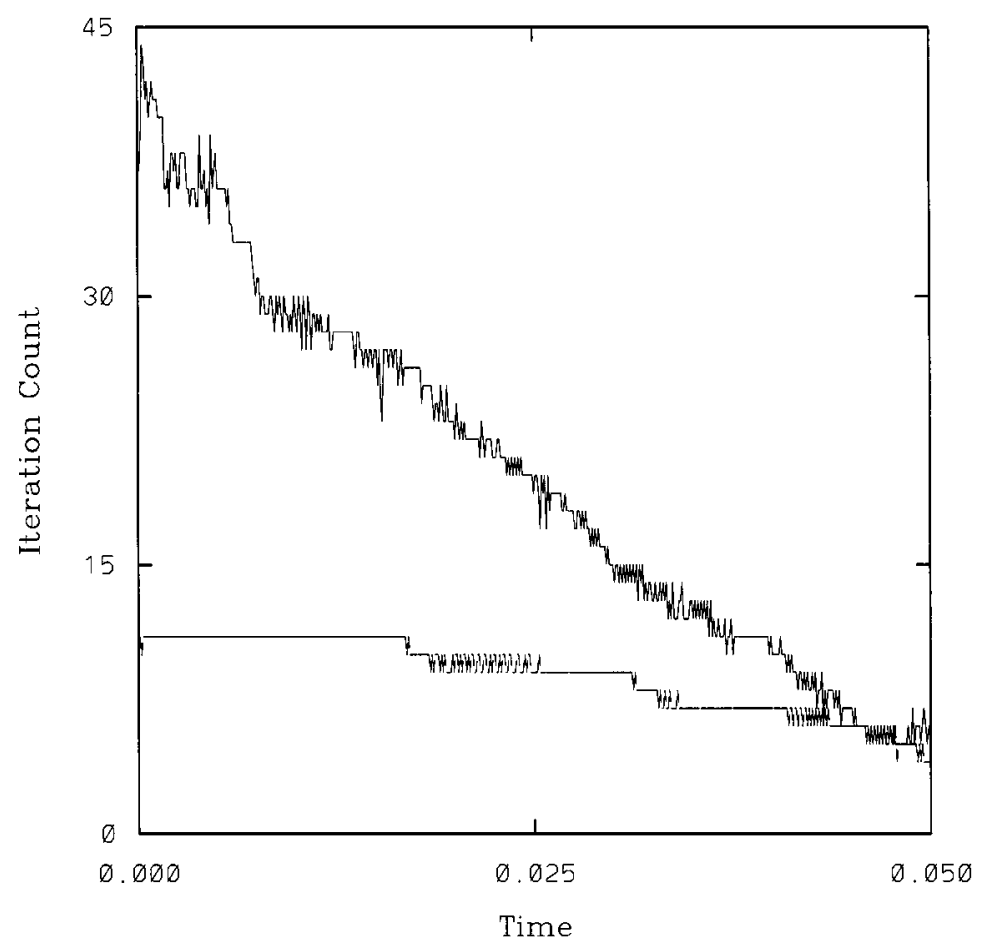

FIG. 1. Iteration count vs time. Dotted line (lower curve), with preconditioners; solid lines (upper curve), without preconditioners. 
to about 4 at the steady state $t=0.05$. In addition, we observe that changing the number of grid points does not change the iteration count if the precipitate shape is well resolved.

\subsection{Performance of Parallel Implementation}

We have implemented our parallel computations on four different machines: (1) an Origin2000 with 195-MHz IP27 Processors; (2) Pentium II cluster with 300-MHz Processors (OS linux 2.0.33) and 100baseT ethernet connection; (3) an IBM-SP2 with 120-MHz P2SC Processors; and (4) a Cray-T3E with 300-MHz DEC alpha Processors. In all cases, we use the Message Passing Interface (MPI) for the communication among processors.

We consider both a three precipitate and a six precipitate calculation. For the Pentium II cluster we also consider a 10 precipitate calculation. In all cases, we take $N=256, Z=2$ for each precipitate and we update in time using Method IF with $\Delta t=10^{-4}$. For the three and six precipitate simulations, some of the precipitates have isotropic surface energy $\left(\tau_{0}=0\right)$ and the others have anisotropic surface energy $\tau_{0}=0.05$ and $\theta_{0}=0$. In the ten precipitate simulation, all precipitates have isotropic surface energy. Because of limited computer resources, we only sample the computational time for the first 20 time steps. We compute the cases on the four different machines and with different number of processors. The speed-up and efficiency are defined in the standard way,

$$
\text { speed-up }=\frac{T_{1}}{T_{P}} \quad \text { efficiency }=\frac{T_{1}}{P T_{P}},
$$

where $T_{P}$ is the computational time using $P$ processors. In Table I, we present the performance results for the Origin-2000 for (a) three precipitates and (b) six precipitates using up to 8 processors. Tables II, IV, and V give the same information for the Pentium II cluster, the IBM-SP, and the Cray-T3E, respectively. We used up to 32 processors for the latter two machines. In Table III, we show the computational time, speed-up, and efficiency for the ten particle simulation on the Pentium II cluster.

Consider first case (a). We see excellent speed-up and efficiency for all four machines using two or four processors. In fact, we see super linear speed-up for the Cray-T3E with two processors. However, with more processors the efficiency decreases and there is almost no improvement in the speed-up from 16 processors to 32 processors. This is because there are not enough operations in computing the summations to compensate for the expense of communicating among the large number of processors. For case (b) where there are more computational points, we see excellent speed-up and efficiency using up to eight processors. Further, at every processor level, both efficiency and speed-up are significantly improved

\section{TABLE I}

\section{Origin-2000}

\begin{tabular}{ccccccc}
\hline Pro & (a) Time (s) & Speed-up & Efficiency & (b) Time (s) & Speed-up & Efficiency \\
\hline 1 & 339 & & & 1343 & & \\
2 & 181 & 1.87 & $94 \%$ & 681 & 1.97 & $99 \%$ \\
4 & 102 & 3.32 & $83 \%$ & 365 & 3.68 & $92 \%$ \\
8 & 63 & 4.46 & $56 \%$ & 213 & 6.30 & $79 \%$ \\
\hline
\end{tabular}

Note. (a) Three precipitates; (b) 6 precipitates; $N=256$ and 20 time-steps. 
TABLE II

Pentium 2 Cluster: 100 BaseT Ethernet Connection

\begin{tabular}{ccccccc}
\hline Pro & (a) Time (s) & Speed-up & Efficiency & (b) Time (s) & Speed-up & Efficiency \\
\hline 1 & 374 & & & 1492 & & \\
2 & 202 & 1.85 & $92 \%$ & 772 & 1.93 & $96 \%$ \\
4 & 124 & 3.02 & $75 \%$ & 421 & 3.59 & $88 \%$ \\
8 & 77 & 4.85 & $47 \%$ & 255 & 5.85 & $73 \%$ \\
16 & 60 & 6.23 & $39 \%$ & 202 & 7.39 & $46 \%$ \\
\hline
\end{tabular}

Note. (a) Three precipitates; (b) 6 precipitates; $N=256$ and 20 time-steps.

\section{TABLE III}

\section{Pentium 2 Cluster: 100 BaseT Ethernet Connection}

\begin{tabular}{rrrr}
\hline Pro & Time (s) & Speed-up & Efficiency \\
\hline 1 & 21017 & & \\
2 & 10738 & 1.95 & $98 \%$ \\
4 & 5666 & 3.70 & $93 \%$ \\
8 & 3071 & 6.84 & $86 \%$ \\
16 & 1573 & 13.36 & $84 \%$ \\
\hline
\end{tabular}

Note. Ten precipitates; $N=256$ and 20 time-steps.

\section{TABLE IV}

IBM-SP

\begin{tabular}{rcccccc}
\hline Pro & (a) Time (s) & Speed-up & Efficiency & (b) Time (s) & Speed-up & Efficiency \\
\hline 1 & 477 & & & 1952 & & \\
2 & 254 & 1.88 & $94 \%$ & 1006 & 1.94 & $97 \%$ \\
4 & 149 & 3.20 & $80 \%$ & 537 & 3.64 & $91 \%$ \\
8 & 92 & 5.18 & $65 \%$ & 309 & 6.32 & $79 \%$ \\
16 & 67 & 7.11 & $44 \%$ & 195 & 10.0 & $63 \%$ \\
32 & 63 & 7.57 & $24 \%$ & 154 & 12.7 & $40 \%$ \\
\hline
\end{tabular}

Note. (a) Three precipitates; (b) 6 precipitates; $N=256$ and 20 time-steps.

TABLE V

\section{Cray-T3E}

\begin{tabular}{rcccccc}
\hline Pro & (a) Time (s) & Speed-up & Efficiency & (b) Time (s) & Speed-up & Efficiency \\
\hline 1 & 224 & & & 897 & & \\
2 & 109 & 2.05 & $1.03 \%$ & 445 & 2.02 & $101 \%$ \\
4 & 57 & 3.93 & $98 \%$ & 227 & 3.95 & $99 \%$ \\
8 & 31 & 7.23 & $90 \%$ & 118 & 7.60 & $95 \%$ \\
16 & 18 & 12.4 & $78 \%$ & 64 & 14.0 & $88 \%$ \\
32 & 18 & 12.4 & $39 \%$ & 46 & 19.5 & $61 \%$ \\
\hline
\end{tabular}

Note. (a) Three precipitates; (b) 6 precipitates; $N=256$ and 20 time-steps. 
over those obtained in case (a). For example, on both the IBM-SP and the the Cray-T3E, the efficiency using 32 processors improves about $60 \%$ from case (a) to case (b). Next, consider the simulation of ten particles on the Pentium II cluster (Table III). Here, excellent speed-up and efficiencies are obtained using up to 16 processors (the most we were able to use). This demonstrates the scalability of our parallel implementation and also suggests that to achieve $80 \%$ efficiency one should use approximately 200 points/processor.

Finally, we notice that among the four machines, the Origin-2000, Pentium II cluster, and IBM-SP have similar efficiency with the Origin-2000 being slightly faster. As expected, the Cray-T3E shows by far the best performance and computational speed of all the machines. It is interesting that the Pentium II cluster, which is by far the most inexpensive machine, gives timings and efficiencies comparable to the much more costly Origin-2000 and IBM-SP.

\subsection{Studies of Different Temporal Schemes}

Four different time-stepping schemes are implemented, three of which ensure that numerical equilibrium and physical equilibrium agree (Methods CN, IF2, and IF3), and one which does not (Method IF). We now elaborate on this result and discuss the performance of the different methods.

We consider first how the difference between the numerical and physical equilibria manifests itself. Figure 2 shows the profiles of a single precipitate at $t=0.2$ with no elasticity, $Z=0$, but with anisotropic surface tension $\tau_{0}=0.05$ and $\theta_{0}=\pi / 4$. The initial condition is a unit circle. Also, $N=512$ and $\Delta t=10^{-4}$. This figure was generated using Method $\mathrm{CN}$, but graphs generated using the other schemes are similar. Note that because of the anisotropic surface energy, the precipitate develops high curvature regions as it approaches

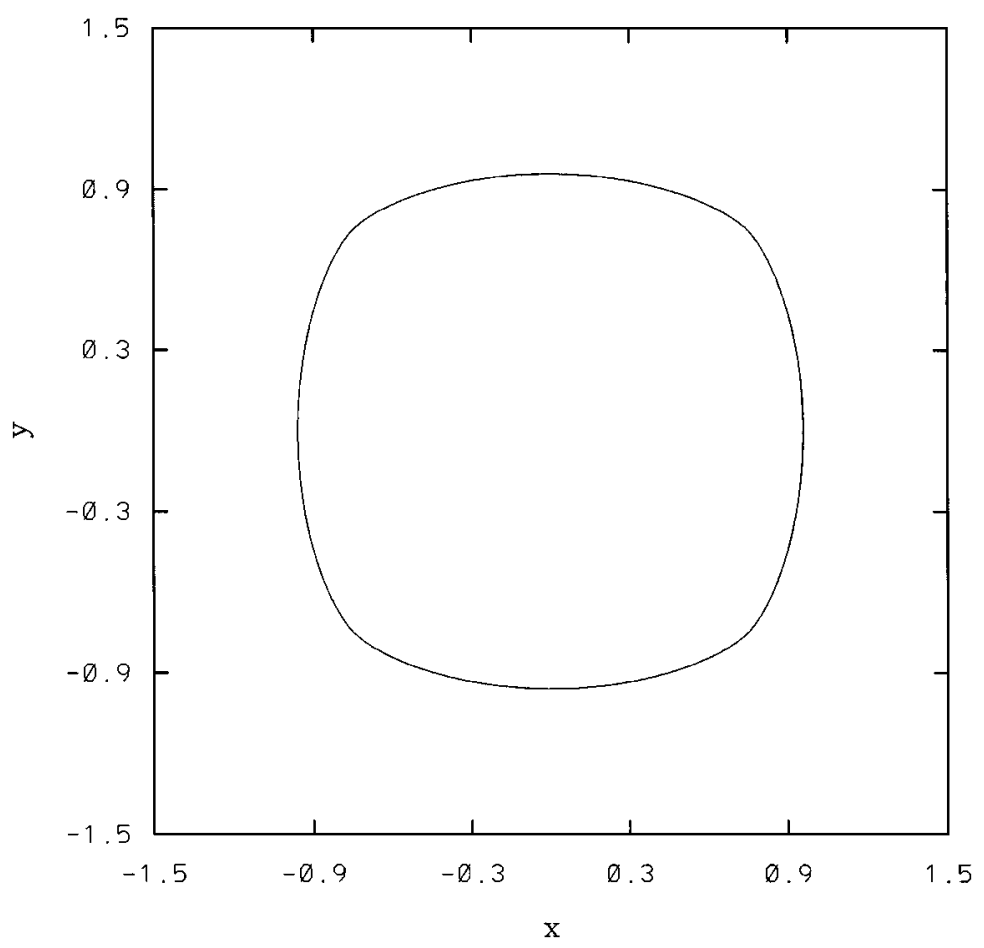

FIG. 2. The profile of one precipitate at $t=0.2$. 


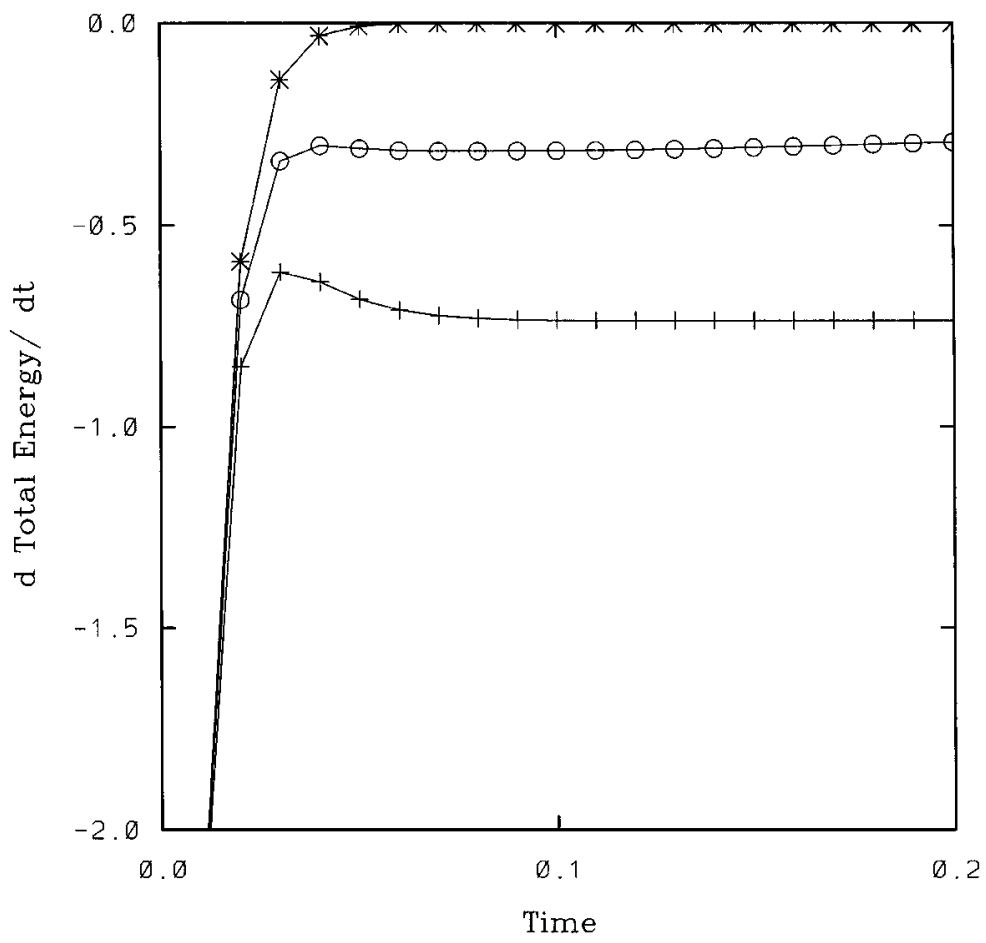

FIG. 3. $d W_{t o t} / d t$ vs time. + , Method IF; $\bigcirc$, Method IF2; $*$, Method CN; $\times$, Method IF3. The $*$ and $\times$ overlap.

the steady-state. By the final time shown, $t=0.2$, the precipitate is very close to its steady state.

Figure 3 shows time derivatives of the total energy plotted against time for computations using Method CN, Method IF, Method IF2, and Method IF3 with the same parameters as above. Clearly, the time derivative of the total energy $\dot{W}_{t o t}$ for the physical system should approach zero as the system approaches the true steady-state. This is indeed the case for Method CN and Method IF3 (whose graphs $*$ and $x$ overlap), but not for Method IF $\left(+\right.$ graph). For Method IF, $\dot{W}_{\text {tot }}$ approaches a nonzero constant $(\approx-0.737)$, indicating that the solution is "trapped" in a numerical steady state. For Method IF2, it appears that $\dot{W}_{t o t}$ is very slowly approaching zero indicating that it may take a very long time before Method IF2 reaches the true equilibrium (as it must).

This behavior is further demonstrated by plotting the composition $c$ on the precipitatematrix interface versus the scaled arclength parameter $\alpha$ at $t=0,0.01,0.02, \ldots, 0.2$ for the four different temporal methods (Fig. 4). In the steady state, $c$ should be constant. Indeed, this is the case for the computations using Method CN and Method IF3 at $t=0.2$. However, for the computations using Method IF and Method IF2, $c$ oscillates in the regions corresponding to the corners of the precipitate. For Method IF2, the amplitude of these oscillations appears to be decreasing very slowly in time indicating a trend towards the true steady state. By comparing the curvatures of the different shapes shown in Fig. 5, one finds that Methods IF and IF2 damp out the high curvature at the corners much more than Methods CN and IF3. This is similar to behavior observed in the diffuse interface calculation of [20], where problems arose due to the high frequencies associated with the diffuse transition layer. 

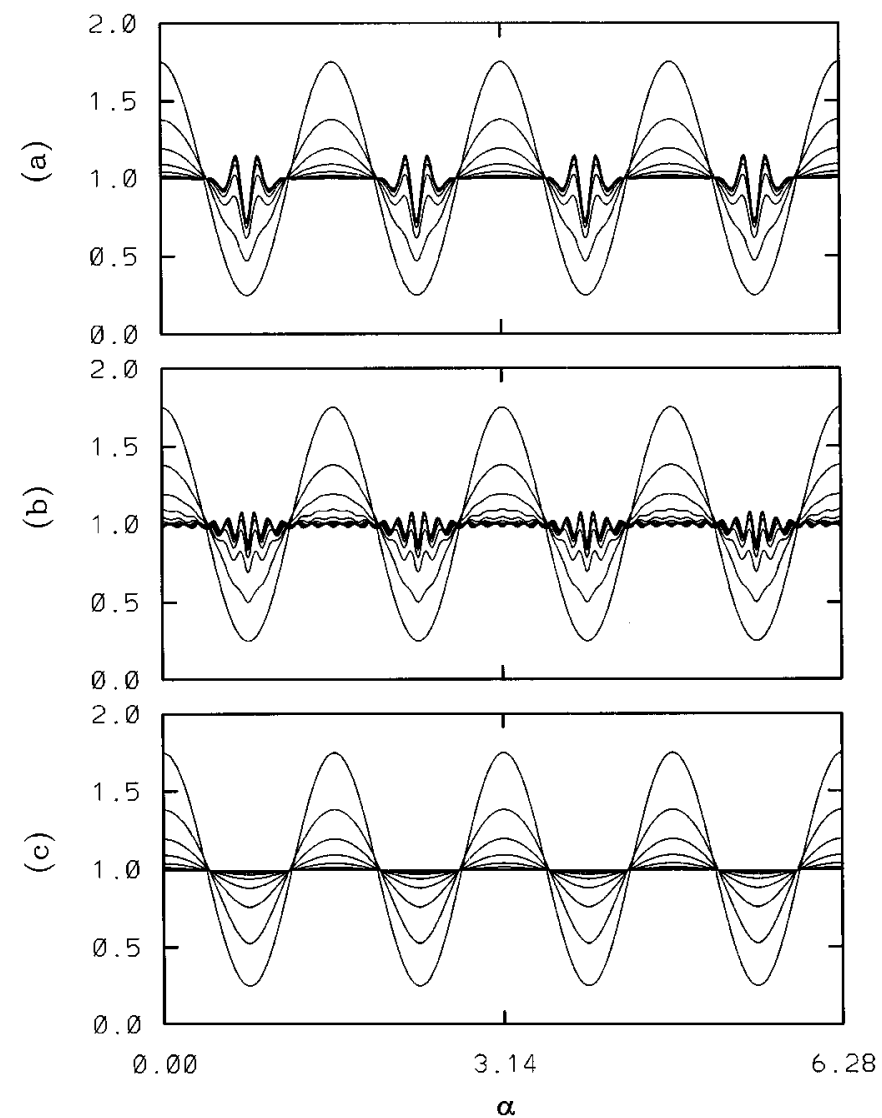

FIG. 4. $c$ at $t=0,0.01,0.02, \ldots, 0.2$. (a) Method IF; (b) Method IF2; (c) Method CN and Method IF3. They overlap.

Essentially, the time stepping used in Methods IF and IF2 acts to filter the numerical solution and in effect prevents the particle from reaching its true equilibrium shape, although we expect that the simulation using Method IF2 will eventually reach the true steady-state at very long times. As $\Delta t$ decreases, Methods IF and IF2 become more accurate albeit more costly. Also, while curves such as Figs. 3 and 4 are striking, the actual differences in the shapes generated by the different methods are negligible.

The problem of damping the high frequencies occurs whenever a particle shape develops corners. Hence it will occur when we include anisotropic elasticity with isotropic surface energy, because the anisotropic elasticity leads to square shapes quite similar to the 4-fold surface energy. Table VI shows results for $Z=8$ and isotropic surface energy $\left(\tau_{0}=0\right)$ computed by using the four temporal schemes using $N=256$ and $\Delta t=2.5 \times 10^{-5}$. Based on the anisotropic surface energy results, the error in the curvature is measured relative to the results computed by using Method CN Table VI shows that the error in curvature is reduced by a factor of 10 by using Method IF 2 over Method IF, and by another factor of 10 by using Method IF3. Also, we see that the time derivative of the total energy, which should be zero in equlibrium, decreases from $10^{-1}$ using Method IF to $10^{-5}$ using either Method $\mathrm{CN}$ or IF3, which is close to the error tolerance of $10^{-6}$ used for solving the elasticity equations.

While Method CN and Method IF3 are good at capturing the true equilibrium of the system, they are also more expensive in terms of computational cost compared to the other 

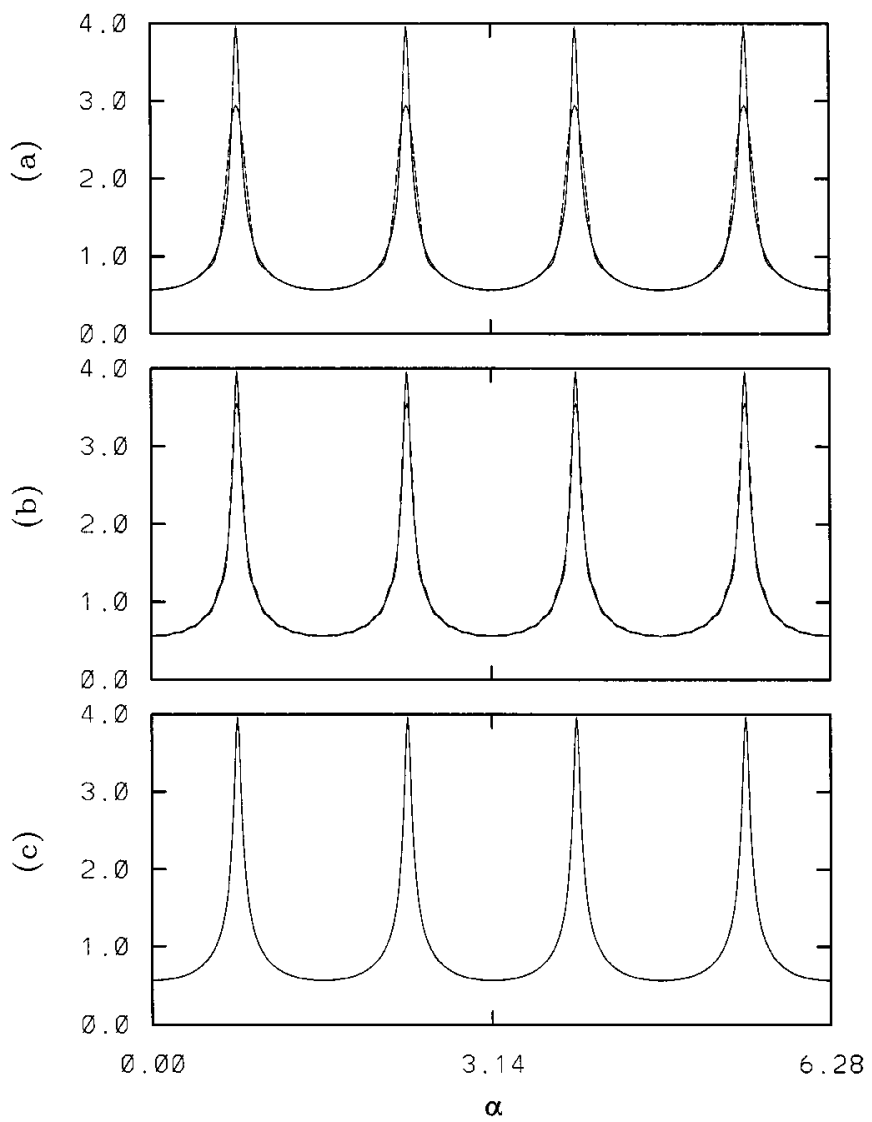

FIG. 5. Curvature vs $\alpha$ at $t=0.2$. Solid curves in all graphs, Method CN; (a) dotted curves, Method IF; (b) dotted curves, Method IF2; (c) dotted curves, Method IF3.

methods. If we use the iteration count per time step as an index of cost, then Method IF and Method IF2 are roughly equivalent, Method IF3 is twice as expensive as Method IF2 and Method CN is twice as expensive as Method IF3. In general, we believe that Method IF3 demonstrates the best combination of accuracy, efficiency, and stability. This is most evident for calculations with large $Z$. For example, with $Z=10$, we are able to use Method IF3 with $N=256$ and $\Delta t=10^{-5}$ to compute the solution such that the the derivative of the energy is about $10^{-5}$ (near steady state). However, for the same $N$ and $\Delta t$, Method $\mathrm{CN}$ diverges because of the accumulation and amplification of the high frequencies in the solutions (primarily due to elastic fields generated by the high curvature regions).

TABLE VI

Comparison of Methods for Cases with $Z=8$

\begin{tabular}{lcccc}
\hline & Method IF & Method IF2 & Method IF3 & Method CN \\
\hline$d W_{\text {tot }} / d t$ at equilibrium $t=0.1$ & $-10^{-1}$ & $-10^{-3}$ & $-10^{-5}$ & $-10^{-5}$ \\
$\begin{array}{l}\text { Maximal curvature difference relative } \\
\text { to the one by Method CN }\end{array}$ & $10^{-1}$ & $10^{-2}$ & $10^{-3}$ & \\
$\begin{array}{l}\text { Average iteration count } \\
\text { per time step }\end{array}$ & 1.6 & 1.7 & 2.9 & 5.3 \\
\hline
\end{tabular}


In summary, Method IF3 has the best balance of accuracy and efficiency of the four methods we have tested. Of the other methods, Method IF and Method IF2 are efficient, but need extremely small time-steps to accurately capture high curvature regions. Method $\mathrm{CN}$ can accurately capture the high curvature regions with a moderate time-step, but it needs a very small time-step for numerical stability in solving the integral equations for elasticity.

\subsection{Convergence Tests}

Analytic solutions for the displacement and traction of a single precipitate in inhomogeneous orthotropic elastic media can be obtained by using conformal mapping techniques; see, for example, [4]. We have verified the static numerical solutions from our boundary integral formulation of the elasticity problem against the analytic solutions in [4]. We have also compared our time-dependent and multi-precipitate computations for the case of homogeneous, cubic elastic media against the solutions found using the elastic solver introduced by Voorhees et al. [43, 41, 42]. The different solution techniques agree up to numerical resolution.

We confirm the numerical accuracy of our results by considering the following problem. We take a single precipitate that is initially a circle of radius unity. The precipitate has dilational misfit $\epsilon_{11}^{T}=\epsilon_{22}^{T}=1, \epsilon_{12}^{T}=0$, there are no applied fields, and the far-field flux $J=0$. The elastic constants are chosen to be cubic in both the matrix and precipitate, with $c_{11}^{M}=c_{22}^{M}=2.03, c_{12}^{M}=1.21, c_{66}^{M}=1, c_{11}^{P}=c_{22}^{P}=3.03, c_{12}^{P}=1.61$, and $c_{66}^{P}=1.35$. Also, $Z=5, \tau_{0}=0.05$, and $\theta_{0}=0$. Time updating is done using Method IF3 and the error tolerance for GMRES is set to $10^{-10}$.

Consider first the resolution in space. We use a computation with $N=1024$ to approximate the exact solution. We compare this solution at time $t=0.1$ to those with $N=64$, 128,256 , and 512. Note that the precipitate has essentially reached its steady state. For all the computations, we choose $\Delta t=2 \times 10^{-3}$. The error is defined to be the largest difference between points on the precipitate-matrix interface. Figure 6 shows the base 10 logarithm of the error plotted against the base 2 logarithm of $N$. For $N=256$, there are 9 digits of accuracy which is close to the GMRES tolerance $10^{-10}$. Consequently, increasing $N$ to $N=512$ does not improve the accuracy. Figure 7 shows similar results for the

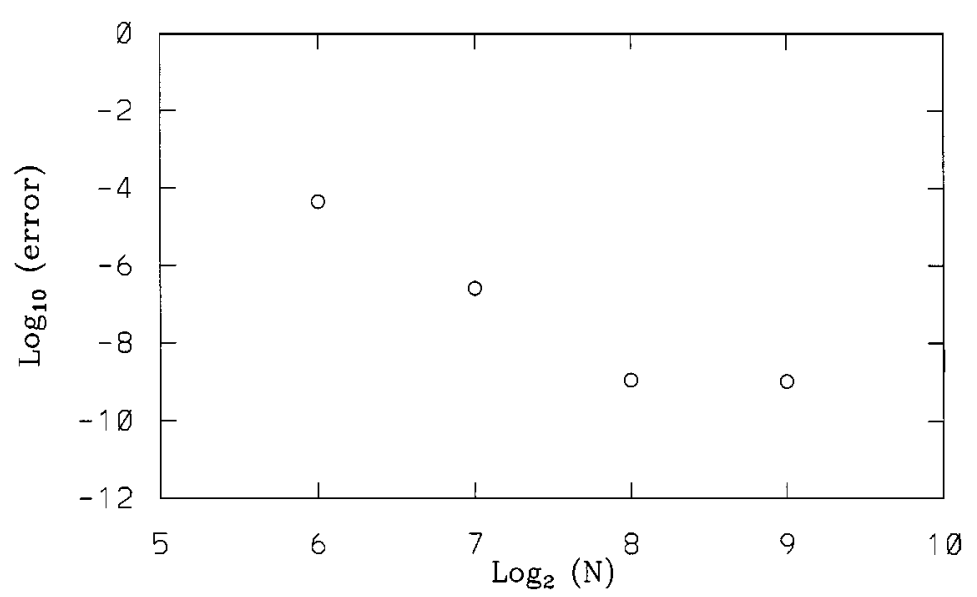

FIG. 6. Resolution study for spatial discretization with the error in the interface location as a function of $N$, $N=64,128,256,512$. 


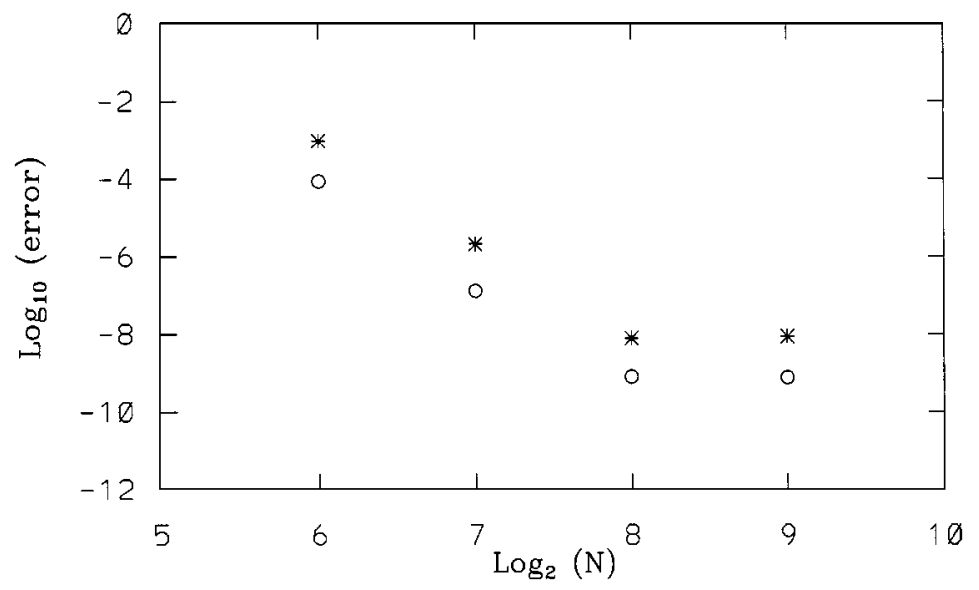

FIG. 7. Resolution study for spatial discretization with the error in the solution of the elasticity equation as a function of $N, N=64,128,256,512$; $\bigcirc$, displacement; $*$, traction.

displacements and tractions on the interface at $t=0.1$. Here the error is taken to be the $l^{2}$ norm of the difference in the appropriate quantity computed for $N$ grid points compared to that with $N=1024$ grid points. We notice that the accuracy for the displacement is about the same as the accuracy of the interface location, while the accuracy for the traction is about one digit worse.

Since $N=256$ is accurate to 9 digits, we use it for the temporal resolution study. We choose the solution with $\Delta t=2 \times 10^{-4}$ to approximate the exact solutions. We compare this to the solution with $\Delta t=2.5 \times 10^{-3}, 1.25 \times 10^{-3}$, and $6.25 \times 10^{-4}$. The error is measured in terms of the interface positions as in Fig. 6. Figure 8 shows the base 10 logarithm of the temporal error plotted against time. The distance between the curves in Fig. 8 uniformly decreases by about a factor of 0.6 with each halving of $\Delta t$ thereby confirming the secondorder accuracy.

It is instructive to consider the temporal resolution for all four time discretization algorithms given in Subsection 3.3. Figure 9 shows the temporal error for all four methods. For Method IF, Method IF2, and Method IF3 the error is measured between the computed

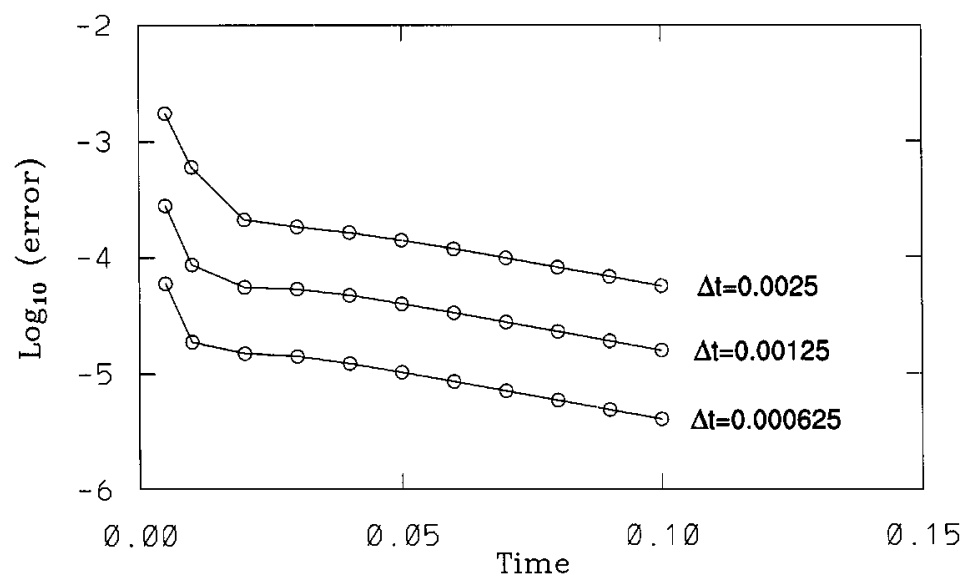

FIG. 8. Resolution study for temporal scheme Method IF3. 


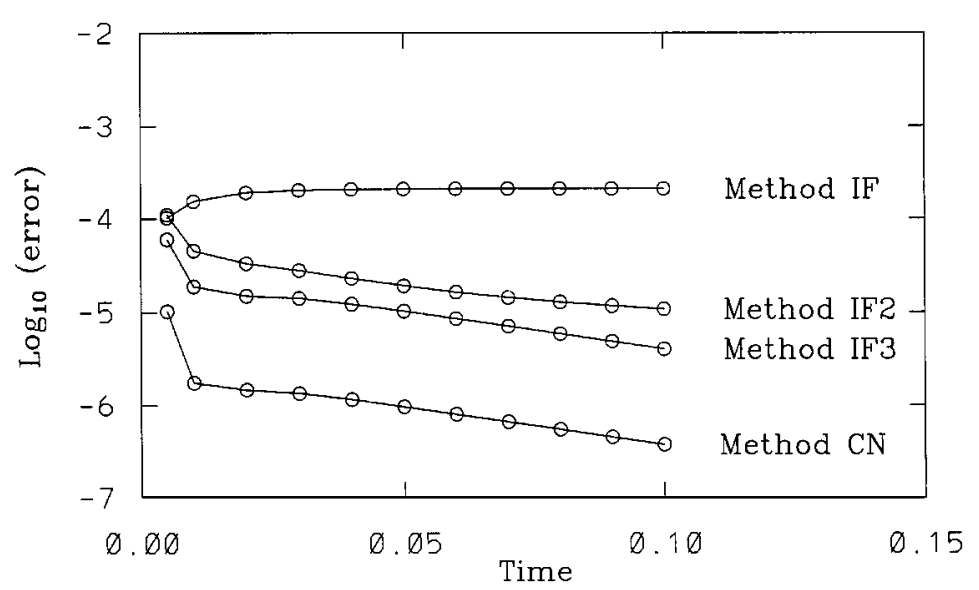

FIG. 9. Error in the interface location vs time for four different methods. Method CN, error between $\Delta t=2 \times 10^{-4}$ and $\Delta t=10^{-4}$ while the other three are error between $\Delta t=6.25 \times 10^{-4}$ and $\Delta t=2 \times 10^{-4}$.

solutions with $\Delta t=6.25 \times 10^{-4}$ and $\Delta t=2 \times 10^{-4}$, while for Method CN the error is measured between the computed solutions with $\Delta t=2 \times 10^{-4}$ and $\Delta t=10^{-4}$. (The reason for choosing a different error measure for Method $\mathrm{CN}$ is that it diverges with $\Delta t=6.25 \times 10^{-4}$.) Note in Fig. 9 that the three methods (IF3, IF2, and CN), which converge to the true steady state, show improved accuracy as the system approaches steady state. In contrast, the error using Method IF saturates in time since since the numerical equilibria depends on the time step $\Delta t$. We remark finally that when the different time steps are taken into account, one can show that Method $\mathrm{CN}$ and Method IF3 have roughly the same level of accuracy.

\section{RESULTS}

We now consider how elastic inhomogeneities and anisotropies as well as surface energy anisotropies affect microstructural development in alloys. We begin by considering Ni-based cubic systems with a $\mathrm{Ni}$ matrix phase and either $\mathrm{Ni}_{3} \mathrm{Al}, \mathrm{Ni}_{3} \mathrm{Ga}$, or $\mathrm{Ni}_{3} \mathrm{Si}$ precipitate phases. Later, we present a calculation using orthotropic elasticity, adapted from observations of melt pockets in mantle rocks.

\subsection{Cubic Elasticity}

We begin with calculations of inhomogeneous cubic elasticity. The elastic constants, normalized by $\mathrm{C}_{66}$ of the matrix Ni phase, are shown in Table VII [32]. For all phases, the anisotropy ratio $A>1$, indicating that the horizontal (i.e., $\langle 10\rangle$ ) and vertical (i.e., $\langle 01\rangle$ ) directions are less stiff than the diagonal (i.e., $\langle 11\rangle$ and $\langle-11\rangle$ ) directions (see [43] for details). Note also that the $\mathrm{Ni}_{3} \mathrm{Al}$ and $\mathrm{Ni}_{3} \mathrm{Ga}$ have elastic constants less than those of the $\mathrm{Ni}$ matrix. Following the notation we used in the isotropic elasticity case [15], we refer to these precipitates as soft. In contrast, the $\mathrm{Ni}_{3} \mathrm{Si}$ precipitates have larger elastic constants than the matrix; we refer to these as hard.

We take an anisotropic surface energy as given by Eq. (2), i.e.,

$$
\tau(\theta)=1+\tau_{0} \cos 4\left(\theta-\theta_{0}\right),
$$

where either $\theta_{0}=0$ or $\theta_{0}=\pi / 4$. When $\theta_{0}=0$, the surface energy has minima along the 
TABLE VII

Elasticity Constants

\begin{tabular}{lcccl}
\hline & $c_{11}$ & $c_{12}$ & $c_{22}$ & $c_{66}$ \\
\hline Nickel & 2.03 & 1.21 & 2.03 & 1.0 \\
$\mathrm{Ni}_{3} \mathrm{Al}$ & 1.83 & 1.21 & 1.83 & 1.02 \\
$\mathrm{Ni}_{3} \mathrm{Ga}$ & 1.54 & .996 & 1.54 & 0.87 \\
$\mathrm{Ni}_{3} \mathrm{Si}$ & 3.04 & 1.62 & 3.04 & 1.35 \\
Olivine & 4.1 & 0.84 & 2.5 & 1.0 \\
Soft precipitate & 1.14 & 0.22 & 1.14 & 0.417 \\
\hline
\end{tabular}

diagonal directions and so the minimum surface energy (Wulff plot) shape will be squarish with sides (facets) oriented perpendicular to the diagonals. See the graphs in Fig. 10 labelled "competing." The reason for this notation will be discussed below. When $\theta_{0}=\pi / 4$, the surface energy has minima along the horizontal and vertical directions and so the Wulff plot shape will be squarish with facets oriented normal to those directions. See the graphs in Fig. 10 labeled "enhanced."

5.1.1. One particle. Consider first the effect of elastic inhomogeneity on the equilibrium shape of a single particle with isotropic surface energy $\left(\tau_{0}=0\right)$. Equilibrium shapes—shapes
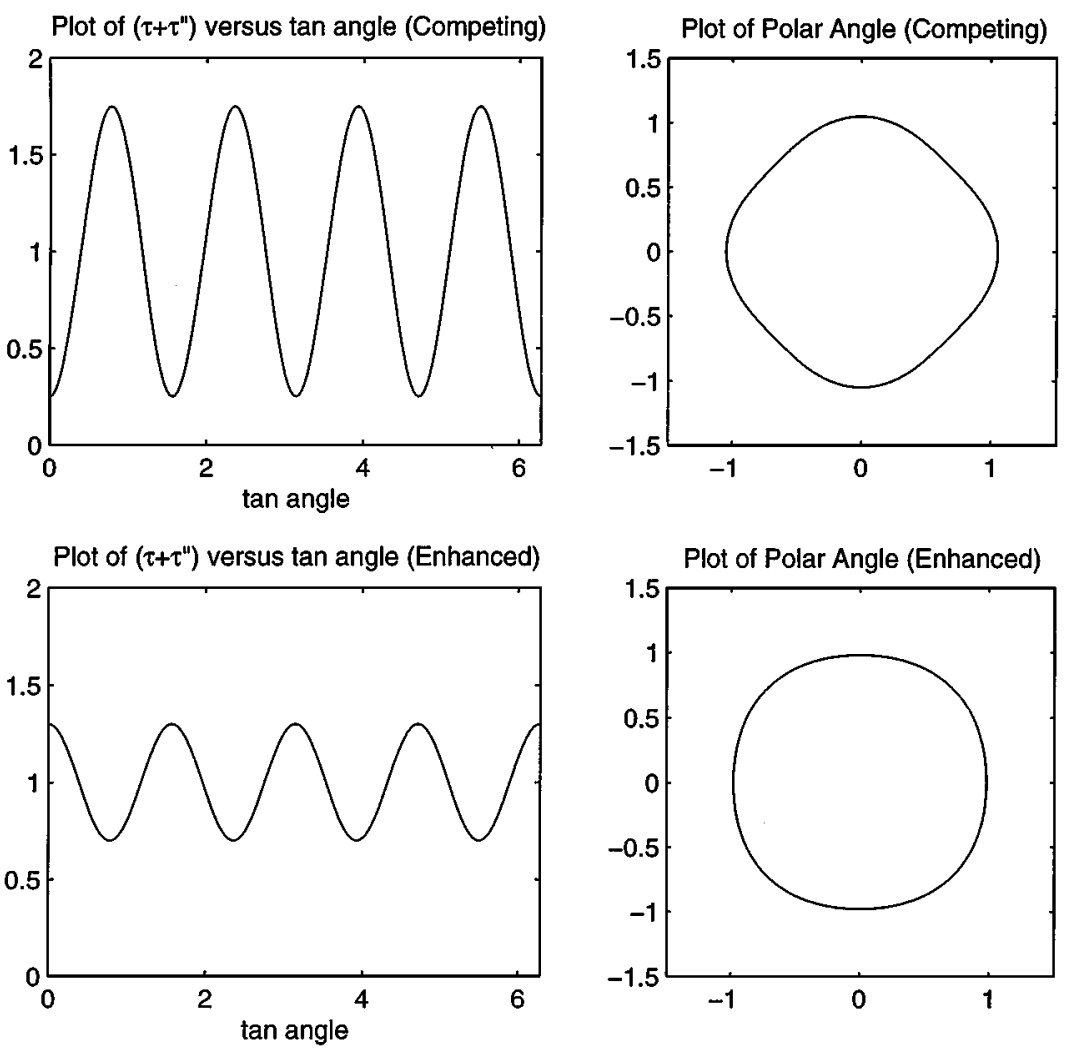

FIG. 10. Anisotropic surface tension. Upper plots, competing; lower plots, enhanced. For each set, left, $\left(\tau+\tau^{\prime \prime}\right)$ vs tangent angle $\theta$; right, polar angle $(\tau \cos (\theta), \tau \sin (\theta)) . \tau=1+\tau_{0} \cos 4\left(\theta-\theta_{0}\right)$. In the competing case, $\tau_{0}=0.05$ and $\theta_{0}=0$. In the enhanced case. $\tau_{0}=0.02$ and $\theta=\pi / 4$. 
for which the chemical potential and composition is uniform along the interface- - have been calculated previously by Thompson et al. for the case of homogeneous elasticity [43], and by Schmidt and Gross [38] for the inhomogeneous case. These workers determined the equilibrium shapes by determining the composition on the interface for a given particle shape, and iterating until the shape giving uniform interfacial composition is found. In contrast, we find the equilibrium shape by evolving an elliptical initial shape until it reaches equilibrium.

Figure 11 shows a series of equilibrium shapes that evolve from the same initial shape. In all these figures, $Z=5$. For the homogeneous system (elastic constants of $\mathrm{Ni}$ for both phases), the equilibrium shape is squarish, in agreement with the calculations of Thompson

Homogeneous

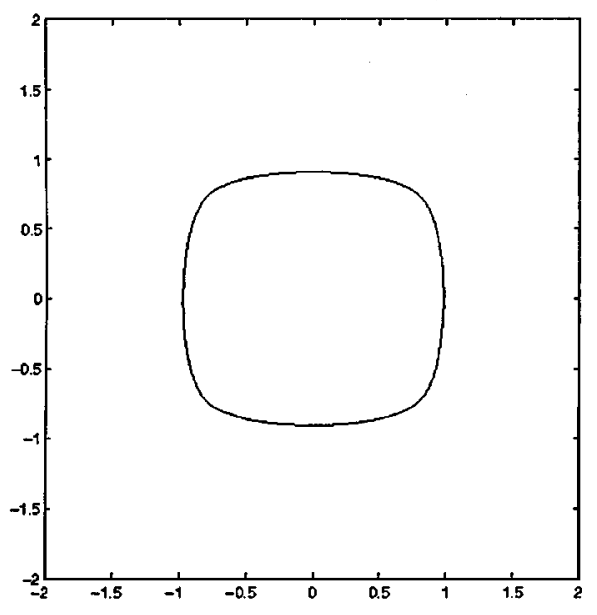

Ni-AI

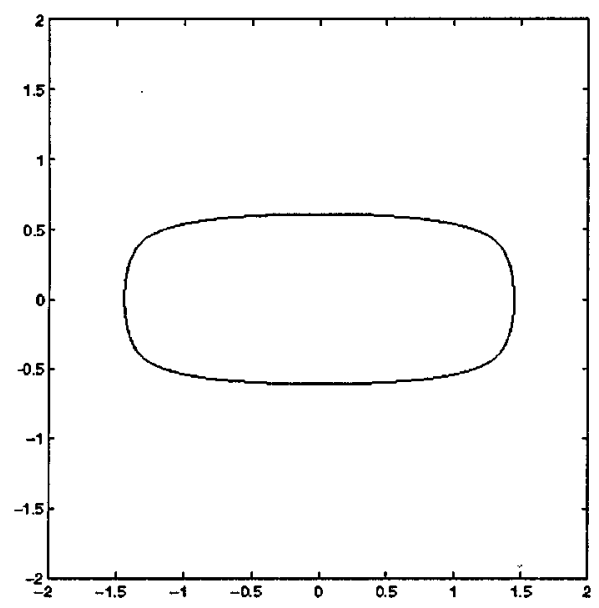

Ni-Si

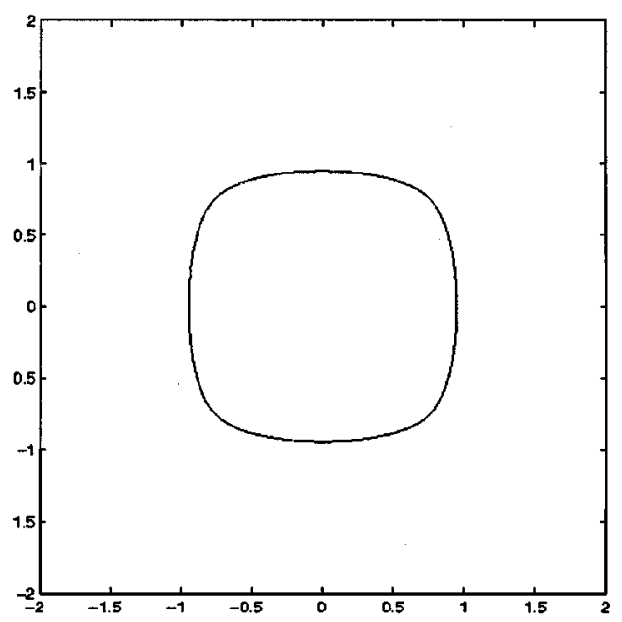

Ni-Ga

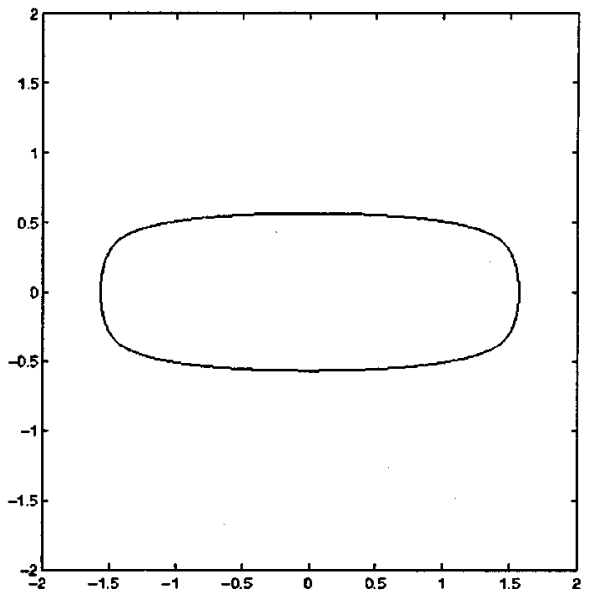

FIG. 11. Equilibrium precipitate shapes, $Z=5, \tau_{0}=0$. For homogeneous simulation, $T=3.5, N=256$, $\Delta t=5 \times 10^{-4}$. For $\mathrm{Ni}_{3} \mathrm{Si}$ simulation, $T=1.3, N=256, \Delta t=1 \times 10^{-4}$. For $\mathrm{Ni}_{3} \mathrm{Al}$ simulation, $T=0.90, N=512$, $\Delta t=1 \times 10^{-4}$. For $\mathrm{Ni}_{3} \mathrm{Ga}$ simulation, $T=1.22, N=512, \Delta t=1 \times 10^{-4}$. In all, the tolerances for the diffusion and the elasticity solvers are $10^{-6}$. 


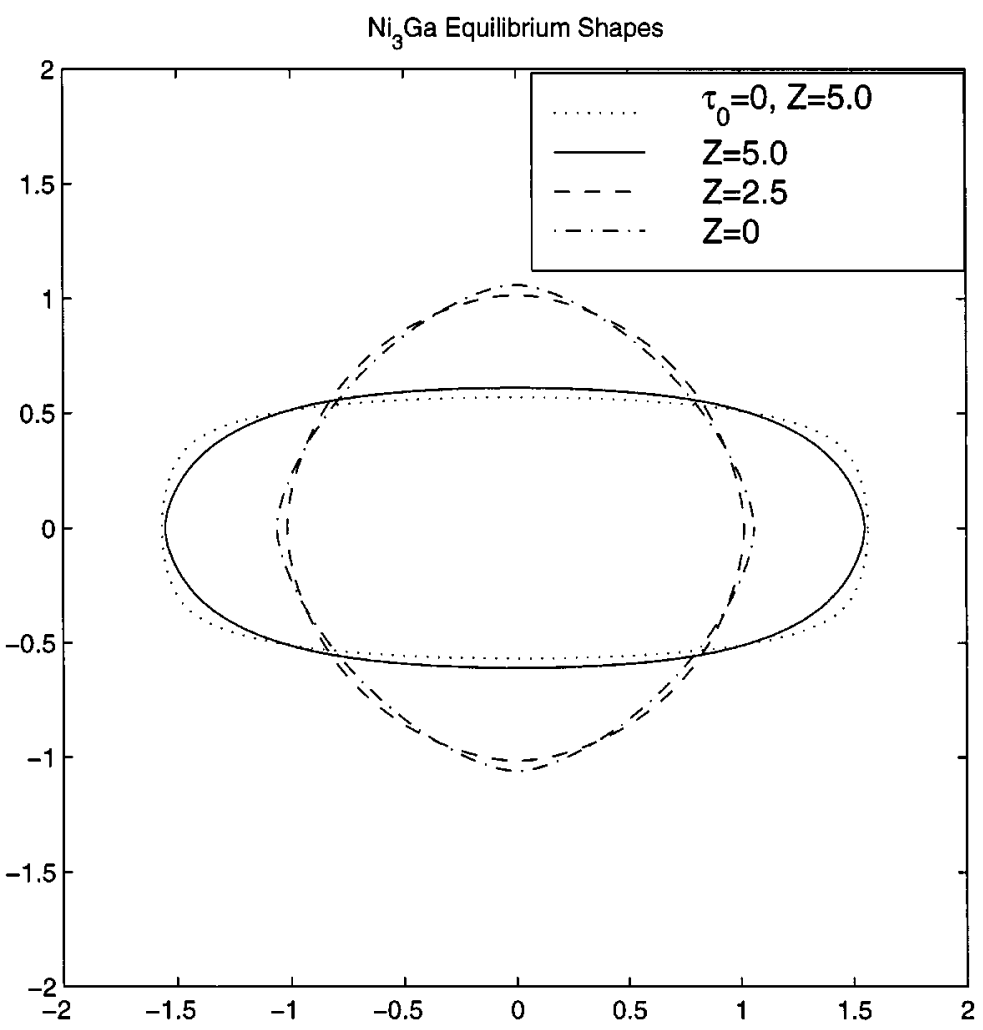

FIG. 12. Equilibrium precipitate shapes for $\mathrm{Ni}_{3} \mathrm{Ga}$ with competing anisotropic surface tension. Dotted curve, $\tau_{0}=0$ (from Fig. 10). In all other curves, $\tau_{0}=0.05$ and $\theta_{0}=0$ (competing surface tension). Solid curve, $Z=5.0$; dashed curve, $Z=2.5$; dot-dashed curve, $Z=0$. For curves with $\tau_{0}=0.05, Z=0$, and $Z=2.5, T=0.1, N=256$, $\Delta t=1 \times 10^{-4}$, and $t o l=10^{-6}$ for both diffusion and elasticity. For curve with $Z=5.0, N=512, \Delta t=2.5 \times 10^{-5}$ with $t o l$ as above.

et al. [43]. ${ }^{4}$ They predict a bifurcation from a squarish shape to a rectangular shape at a value $Z=5.6$. Based on calculations using isotropic elasticity, see, e.g., [15, 14] as well as the results of Schmidt and Gross [38], one expects this bifurcation point to be higher for hard precipitates and lower for soft precipitates. This agrees with our results. The equilibrium $\mathrm{Ni}_{3} \mathrm{Si}$ precipitate is squarish for $Z=5$, while the equilibrium $\mathrm{Ni}_{3} \mathrm{Al}$ and $\mathrm{Ni}_{3} \mathrm{Ga}$ precipitates both have rectangular shapes, with the "softer" $\mathrm{Ni}_{3} \mathrm{Ga}$ precipitate having the larger aspect ratio. While these trends are expected, it is surprising to note the effect of the small inhomogeneity of the $\mathrm{Ni}-\mathrm{Ni}_{3} \mathrm{Al}$ system (see Table VII) on the equilibrium shape. Indeed, one of our qualitative observations throughout this study is that small elastic inhomogeneities in anisotropic systems affect microstructure much more profoundly than similar inhomogeneities in isotropic systems.

We now consider how anisotropic surface energy interacts with elasticity in producing an equilibrium shape. We first take $\tau_{0}=0.05$ and $\tau_{0}=0$. In this case, the Wulff shape $(Z=0)$ has facets oriented at $45^{\circ}$ to the facets of the elastic equilibrium shapes in Fig. 11. Therefore, the surface energy anisotropy "competes" with the elastic anisotropy.

In Fig. 12, equilibrium shapes of a $\mathrm{Ni}_{3} \mathrm{Ga}$ precipitate (soft) are shown for different values of $Z$. The $\tau_{0}=0$ shape (from Fig. 11) is also superposed on Fig. 12 for comparison. One 


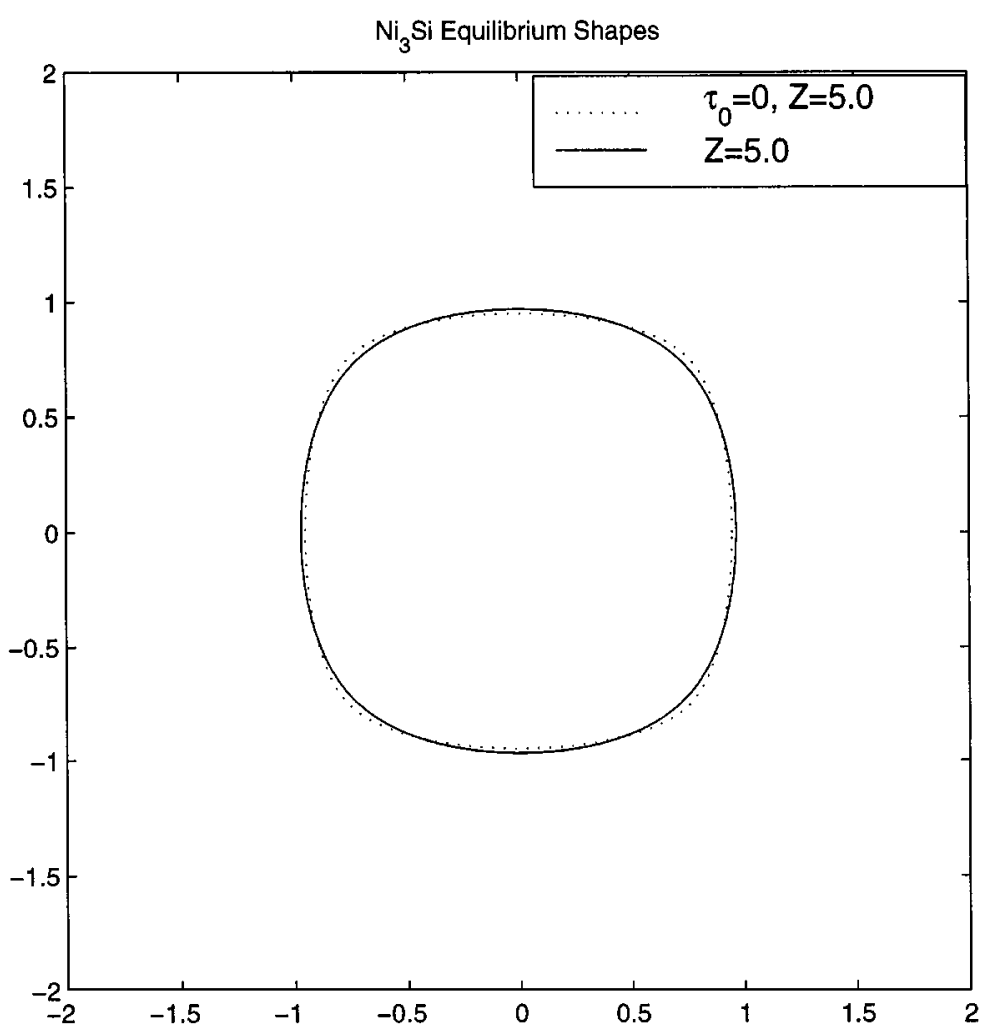

FIG. 13. Equilibrium precipitate shapes for $\mathrm{Ni}_{3} \mathrm{Si}$. Dotted curve, $\tau_{0}=0$ (from Fig. 10). Solid curve, $\tau_{0}=0.05$, $\theta_{0}=0, Z=5, T=0.10, N=256$, and $\Delta t=1 \times 10^{-4}$. In all simulations, $t o l=10^{-6}$ for both diffusion and elasticity.

observes that the anisotropic surface energy causes the $Z=5$ precipitate to become more ellipsoidal compared to the corresponding precipitate with isotropic surface tension. The aspect ratios of these two shapes are quite similar indicating that for this $\tau_{0}$, the value of $Z$, at which the bifurcation from a squarish to rectangular shape occurs, seems to be unaffected by the anisotropic surface energy. When $Z=0$, one recovers the Wulff shape seen in Fig. 10. As $Z$ increases, the facets change orientation such that by $Z=5$, we observe horizontal facets consistent with the elastic equilibrium shapes shown in Fig. 11 although the vertical facets seem to disappear.

In Fig. 13, equilibrium shapes of a $\mathrm{Ni}_{3} \mathrm{Si}$ precipitate (hard) are shown for $Z=5$ both with and without anisotropic surface energy. We observe little difference in the overall shapes, although as expected, the competing anisotropic surface energy acts to smooth the corners.

We next consider a surface energy anisotropy with $\theta_{0}=\pi / 4$. Here, the facets in both the Wulff and elastic equilibrium shapes are aligned horizontally and vertically. Therefore, this surface energy "enhances" the elastic anisotropy. In this case, we had difficulty computing with $\tau_{0}=0.05$ and so we used the smaller value $\tau_{0}=0.02$. The results are shown in Fig. 14 for an $\mathrm{Ni}_{3} \mathrm{Si}$ precipitate. Analogous results are obtained for $\mathrm{Ni}_{3} \mathrm{Ga}$ and are not shown. In Fig. 14, $Z=5$ and precipitates with and without anisotropic surface energy are shown. We observe that the anisotropic surface energy has little effect on the overall shapes although the anisotropic case has slightly sharper corners. 


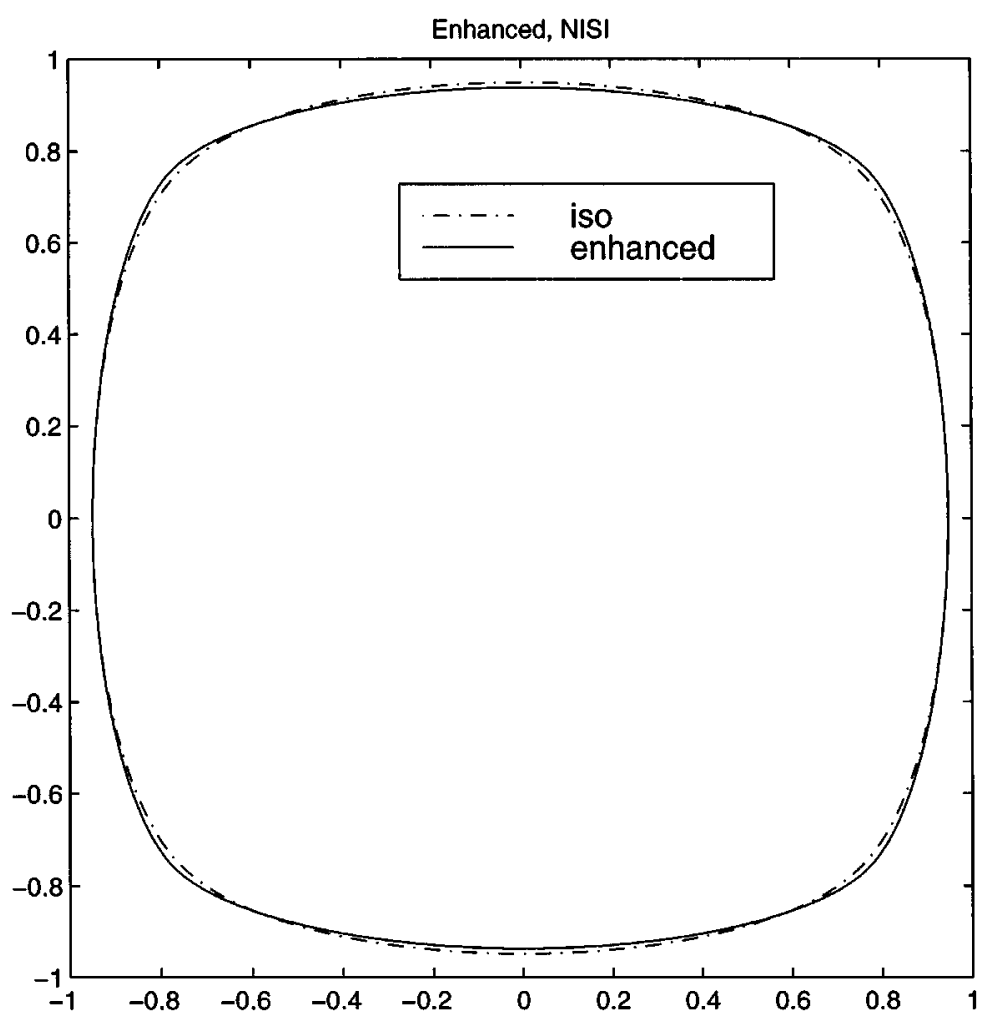

FIG. 14. Equilibrium precipitate shape for $\mathrm{Ni}_{3} \mathrm{Si}$ with enhanced anisotropic surface tension. Dotted curve, $\tau_{0}=0$ (from Fig. 10). Solid curve, $\tau_{0}=0.02, \theta_{0}=\pi / 4, Z=5, T=1.0, N=256$, and $\Delta t=1 \times 10^{-4}$. In all simulations, $t o l=10^{-6}$ for both diffusion and elasticity.

5.1.2. Two particles. We now consider the evolution of two particles with isotropic surface energy. The primary focus of our simulations is to test how inhomogeneity affects the observation of a a stable interparticle spacing in elastically homogeneous systems [42]. The presence of this stable spacing has been explained by Su and Voorhees [42], who used the idea of configurational forces [10] to account for the interaction between particles. However, the calculation of configurational forces breaks down when the elastic constants of the precipitate and matrix phases differ. In addition, based on the results for inhomogeneous, isotropic elasticity $[15,38]$, we expect inhomogeneity to have a strong influence on interparticle forces.

Figure 15 shows the evolution of two particles for the different systems we consider. The initial configuration in all cases consists of two unit circles separated by a distance of 1 unit. Also, $Z=5$ for all cases. For the homogeneous case and the case with $\mathrm{Ni}_{3} \mathrm{Si}$ particles, the final times correspond to when particle evolution essentially ceased. For the cases with $\mathrm{Ni}_{3} \mathrm{Al}$ and $\mathrm{Ni}_{3} \mathrm{Ga}$ particles, the final times correspond to when the interparticle spacing was too small to resolve the interfaces for the numerical parameters used.

We observe that the spacing between the hard $\mathrm{Ni}_{3} \mathrm{Si}$ particles is larger than the spacing between the homogeneous particles, while the spacing between the soft $\mathrm{Ni}_{3} \mathrm{Al}$ and $\mathrm{Ni}_{3} \mathrm{Ga}$ particles is smaller than the spacing between the homogeneous particles. This is consistent with simulations in isotropic media, which indicate that hard particles repel, while soft particles attract. However, the situation is more complex with anisotropic elasticity. 
Homogeneous

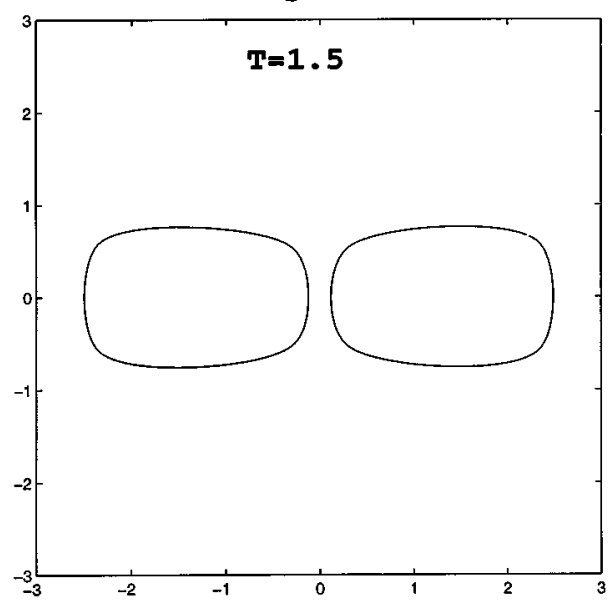

Ni-Si

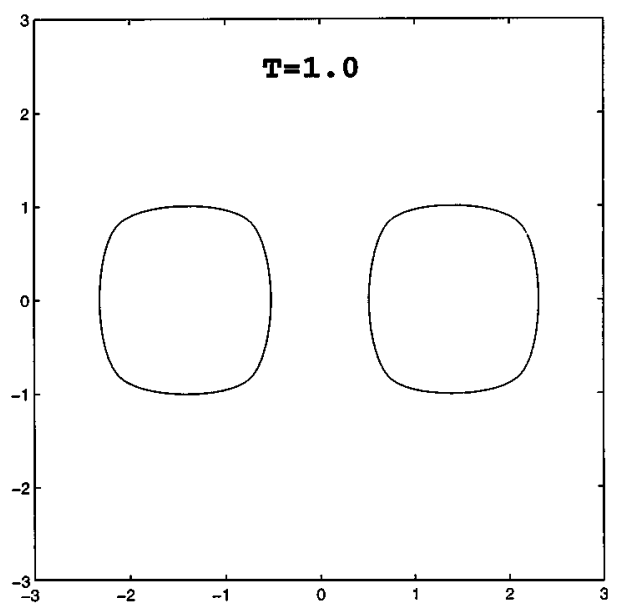

$\mathbf{N i}-\mathbf{G a}$

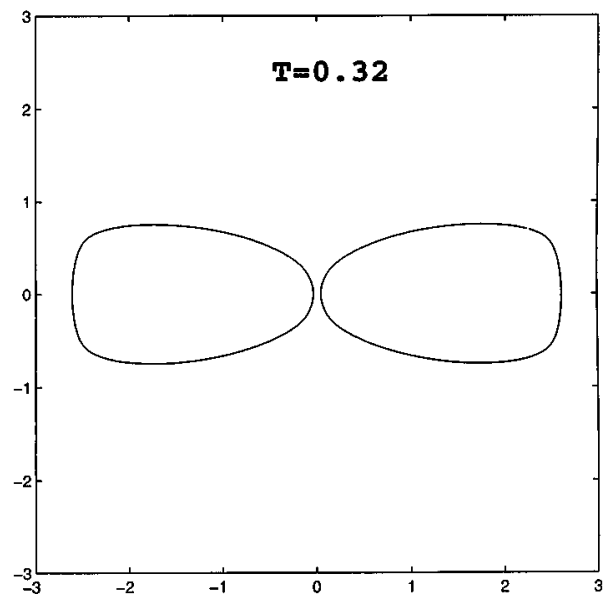

Ni-A1

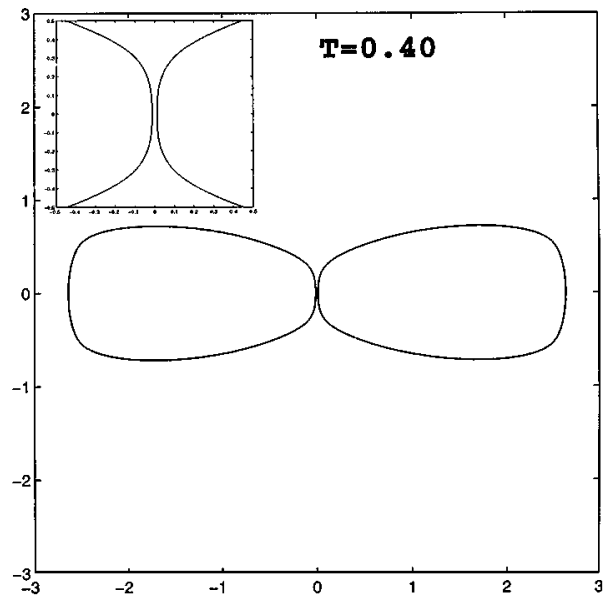

FIG. 15. Two precipitate simulations: shapes. Isotropic surface tension $\tau_{0}=0, Z=5.0$. For homogeneous simulation, $N=256$ on each precipitate, $\Delta t=1 \times 10^{-4}$. For $\mathrm{Ni}_{3} \mathrm{Ga}$ simulation, $N=512$ on each precipitate, $\Delta t=5 \times 10^{-5}$. For $\mathrm{Ni}_{3} \mathrm{Si}$ simulation, $N=256$ on each precipitate, $\Delta t=1 \times 10^{-4}$. For $\mathrm{Ni}_{3} \mathrm{Al}$ simulation, $N=512$ and $\Delta t=5 \times 10^{-5}$ initially, for $t \geq 0.36, N=1024$, and $t=2.5 \times 10^{-5}$. In all simulations, tol $=10^{-6}$ for both diffusion and elasticity.

Figure 16 shows the velocity of the point of closest approach versus the half-distance between the particles. The initial condition corresponds to a half-distance of 0.5 . In all cases, the particles initially repel (velocity $>0$ ) as the particles square off. At later times, the particles move towards each other (velocity $<0$ ) though the details depend on the elastic inhomogeneity. In the homogeneous and the $\mathrm{Ni}_{3} \mathrm{Si}$ case, the particles move towards each other with very small velocities which seemingly tend to zero at a finite interparticle distance.

The behavior observed in the $\mathrm{Ni}_{3} \mathrm{Al}$ and $\mathrm{Ni}_{3} \mathrm{Ga}$ systems is more interesting. In these cases, the interparticle attraction at later times is much stronger and may lead to particle merging. In the $\mathrm{Ni}_{3} \mathrm{Ga}$ case, the interparticle velocity becomes increasingly negative as the interparticle spacing decreases. This suggests the particles will merge. In the $\mathrm{Ni}_{3} \mathrm{Al}$ 

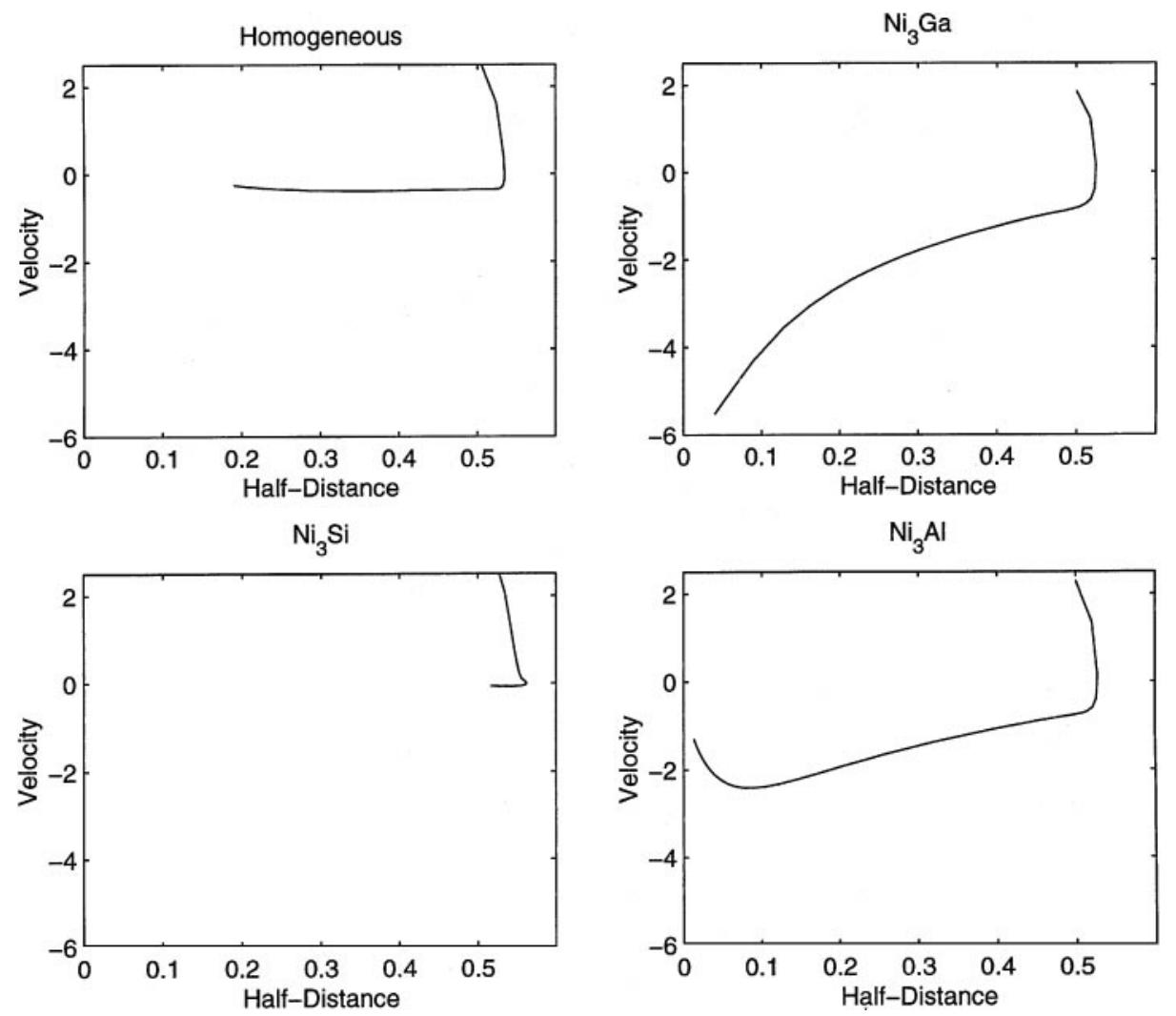

FIG. 16. Two precipitate simulations: velocity. Velocity of $\mathrm{Pt}$ of closest approach vs min half-distance for approaching precipitates in previous Fig. 15 . For $\mathrm{Ni}_{3} \mathrm{Si}$, velocity of centroid of right particle vs $x$-component of centroid of right particle.

case (which is nearly homogeneous), however, there is a turning point in the velocity at small separations although the velocity remains negative. More refined calculations are necessary to determine whether there is in fact a small nonzero interparticle spacing in this case. The difference between the $\mathrm{Ni}_{3} \mathrm{Ga}$ and $\mathrm{Ni}_{3} \mathrm{Al}$ cases is also reflected in the particle shapes in the interparticle region (Fig. 15); in the $\mathrm{Ni}_{3} \mathrm{Ga}$ case, the particles appear to curved towards each other while in the $\mathrm{Ni}_{3} \mathrm{Al}$ case the two particles have "squared off" against each other.

To conclude this section, and to indicate how sensitive the above results are to changes in the physics of the problem, we consider one example with anistropic surface energy. We take two $\mathrm{Ni}_{3} \mathrm{Al}$ precipitates with "competing" anisotropic surface energies $\tau_{0}=0.05$ and $\theta_{0}=0$. All other choices are as above. Figure 17 shows the precipitates at $T=0.27$. Observe that unlike the isotropic surface energy case with $\mathrm{Ni}_{3} \mathrm{Al}$ precipitates shown in Fig. 15, the particles are curved towards each other in the interaction region, consistent with the Wulff shape of each particle. Moreover, as seen in the inset, there is a much larger interparticle attraction in this case compared to the isotropic surface energy case; the velocity becomes more negative as the interparticle spacing decreases. Thus, we expect that in this situation, the particles will merge. Considering the same example with "enhancing" anisotropic surface energy with $\tau_{0}=0.02$ and $\theta_{0}=\pi / 4$, we find less particle attraction than in either the isotropic or competing surface tension cases. This is seen in Fig. 18. Despite 
$\mathrm{Ni}_{3} \mathrm{Al}$ with $\tau_{0}=0.05$ (Competing)

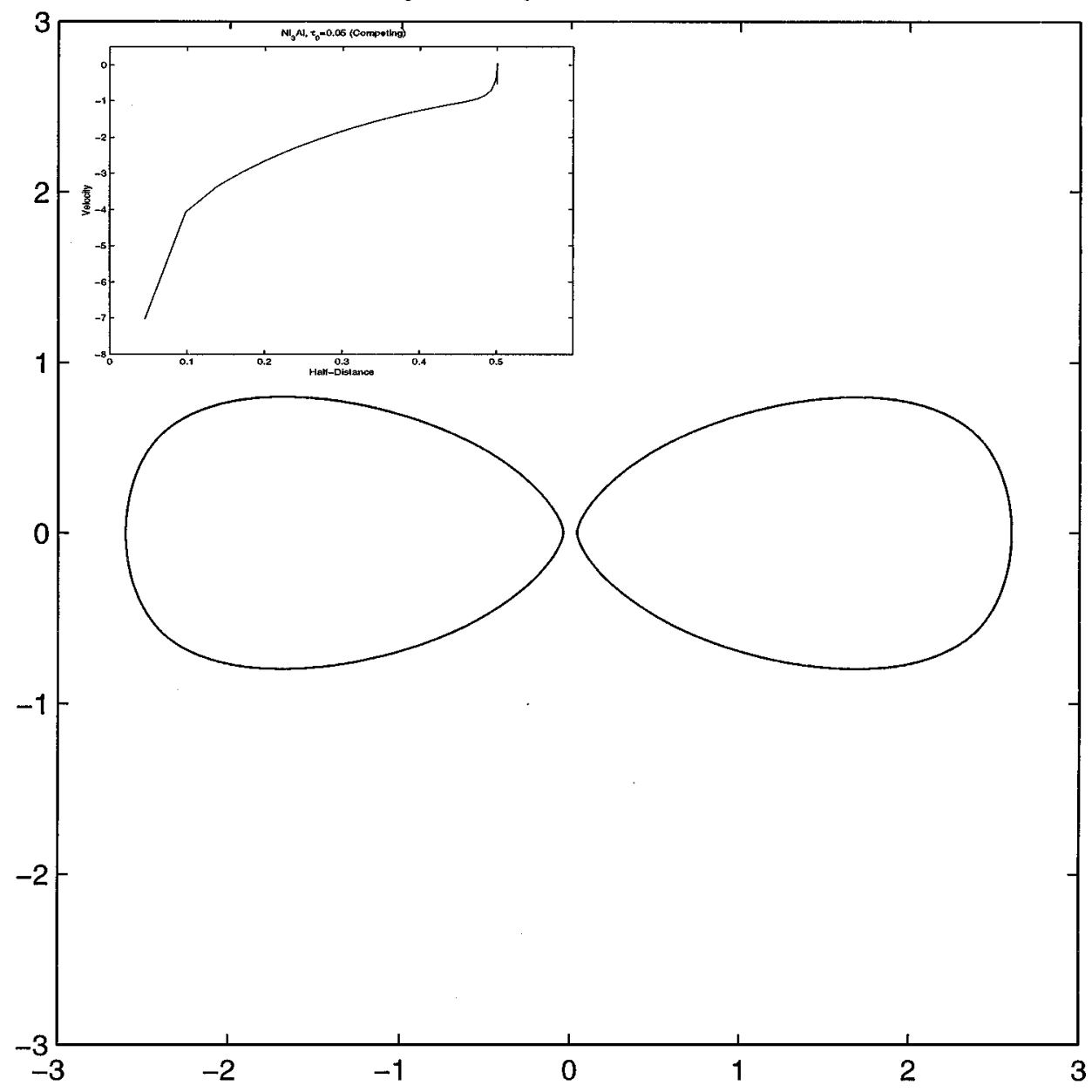

FIG. 17. Two precipitate simulation $\mathrm{Ni}_{3} \mathrm{Al}$, shape at $T=0.27$ with velocity vs half-distance inset. Anisotropic

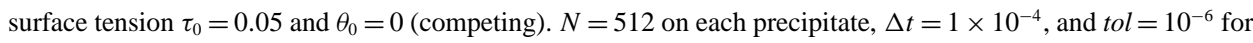
both diffusion and elasticity.

the lowering of attractive forces in this case, it is still unclear whether particle merger will be avoided.

5.1.3. Multiple particles. We next consider the coarsening of systems with multiple precipitates. We investigate two cases of precipitates embedded in a $\mathrm{Ni}$ matrix: (a) 10 $\mathrm{Ni}_{3} \mathrm{Al}$ (soft) precipitates and (b) $10 \mathrm{Ni}_{3} \mathrm{Si}$ (hard) precipitates. In both cases, we take $Z=1$, $\Delta t=5 \times 10^{-4}, N=256$ for each precipitate, the diffusion tolerance $10^{-10}$, the elasticity tolerance $10^{-5}$, and the initial precipitates are all circular. We choose the elastic tolerance to be $10^{-5}$ in order to reduce the computational cost of our simulations.

Because our boundary integral formulation breaks down when topological transitions such as particle vanishing occurs, we remove precipitates by hand when their area decreases below 0.1 . In a prior work [15], the effects of this procedure were carefully investigated in the context of isotropic, inhomogeneous elasticity. It was found that the energy $W_{t o t}$ and the envelope of the time derivative of the energy $\dot{W}_{t o t}$ are continuous through precipitate 


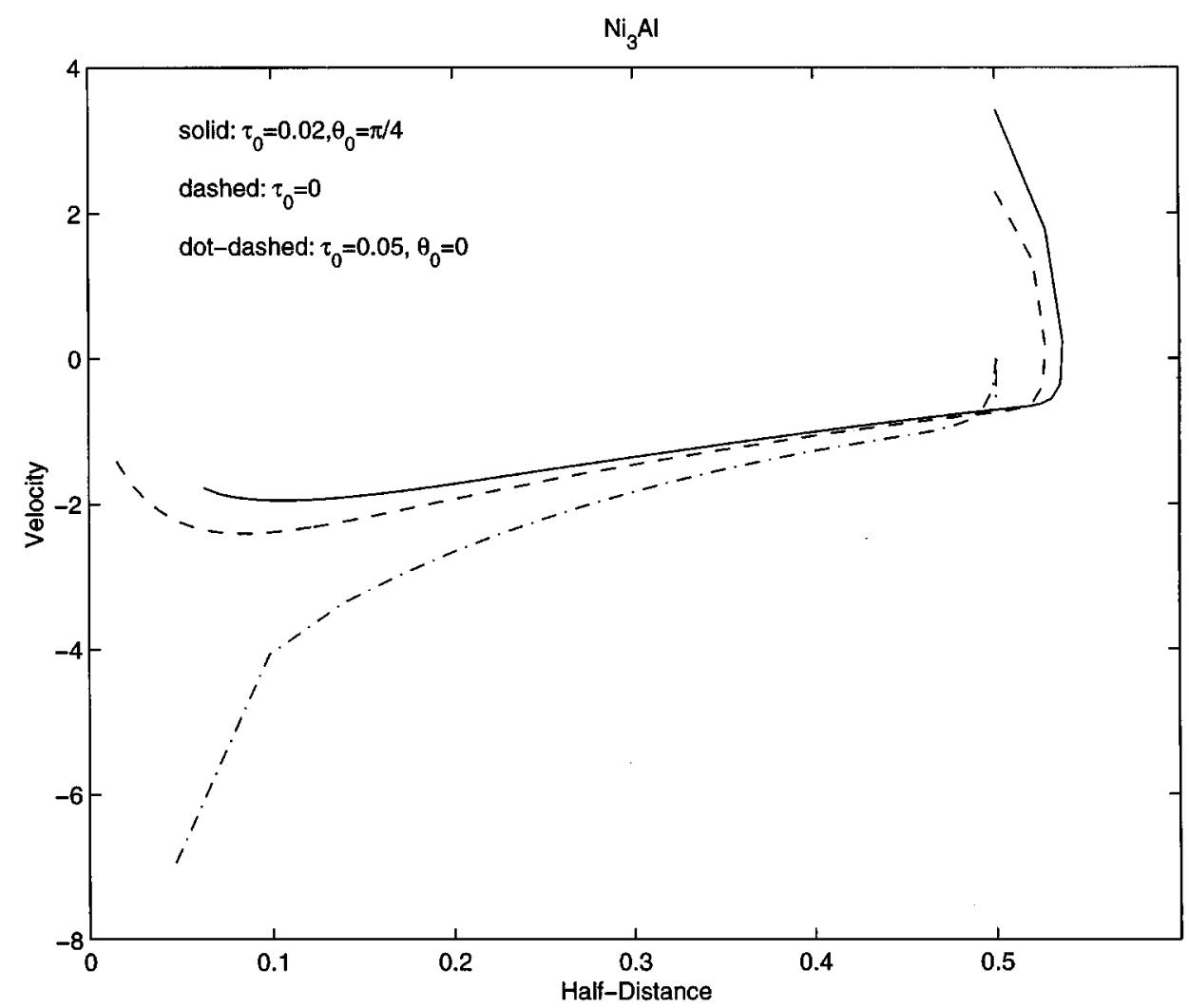

FIG. 18. Two precipitate simulation $\mathrm{Ni}_{3} \mathrm{Al}$, velocity vs half-distance. Solid curve, $\tau_{0}=0.02$ and $\theta_{0}=\pi / 4$ (enhanced); $N=512$ and $\Delta t=1 \times 10^{-4}$ up to $t=0.40, N=1024$, and $\Delta t 5 \times 10^{-5}$ for $0.40<t \leq 0.42(t=0.42$ is final time shown); $t o l=10^{-6}$ for both diffusion and elasticity. Dashed curve, $\tau_{0}=0.0$ from Fig. 16 . Dot-dashed curve, $\tau_{0}=0.05, \theta_{0}=0$ from Fig. 17 .

vanishing. $\dot{W}_{\text {tot }}$ itself, however, diverges negatively as precipitates vanish. Moreover, as the precipitates vanish, they become more circular due to the dominance of the surface energy over the elastic energy. Although we do not present them, we find these results still hold true for the simulations of $\mathrm{Ni}_{3} \mathrm{Al}$ and $\mathrm{Ni}_{3} \mathrm{Si}$ precipitates presented below.

The evolution of systems (a) and (b) in time is shown in Fig. 19. The solid curves correspond to $\mathrm{Ni}_{3} \mathrm{Al}$ precipitates and the dashed curves correspond to the $\mathrm{Ni}_{3} \mathrm{Si}$ precipitates. Note that only the $\mathrm{Ni}_{3} \mathrm{Si}$ precipitates are shown at time $t=20.09$ for reasons explained below. From a macroscopic point of view, there seems to be little difference in the results of the two simulations over the times considered. The precipitates become squarish at very early times and there is only a small amount of particle translation. One can observe that the upper and lower two relatively large pairs of precipitates tend to align along the horizontal direction locally. The global alignment of all precipitates on the horizontal and vertical directions appears to occur on a longer time scale. On the time scale presented, the kinetics appears to be primarily driven by the surface energy which favors coarsening-the growth of large precipitates at the expense of the small precipitates to reduce the surface energy.

Upon closer examination, differences between the simulations are observed. For example, consider the result at time $t=15.77$ which is shown in Fig. 20. In the $\mathrm{Ni}_{3} \mathrm{Al}$ case, the two 

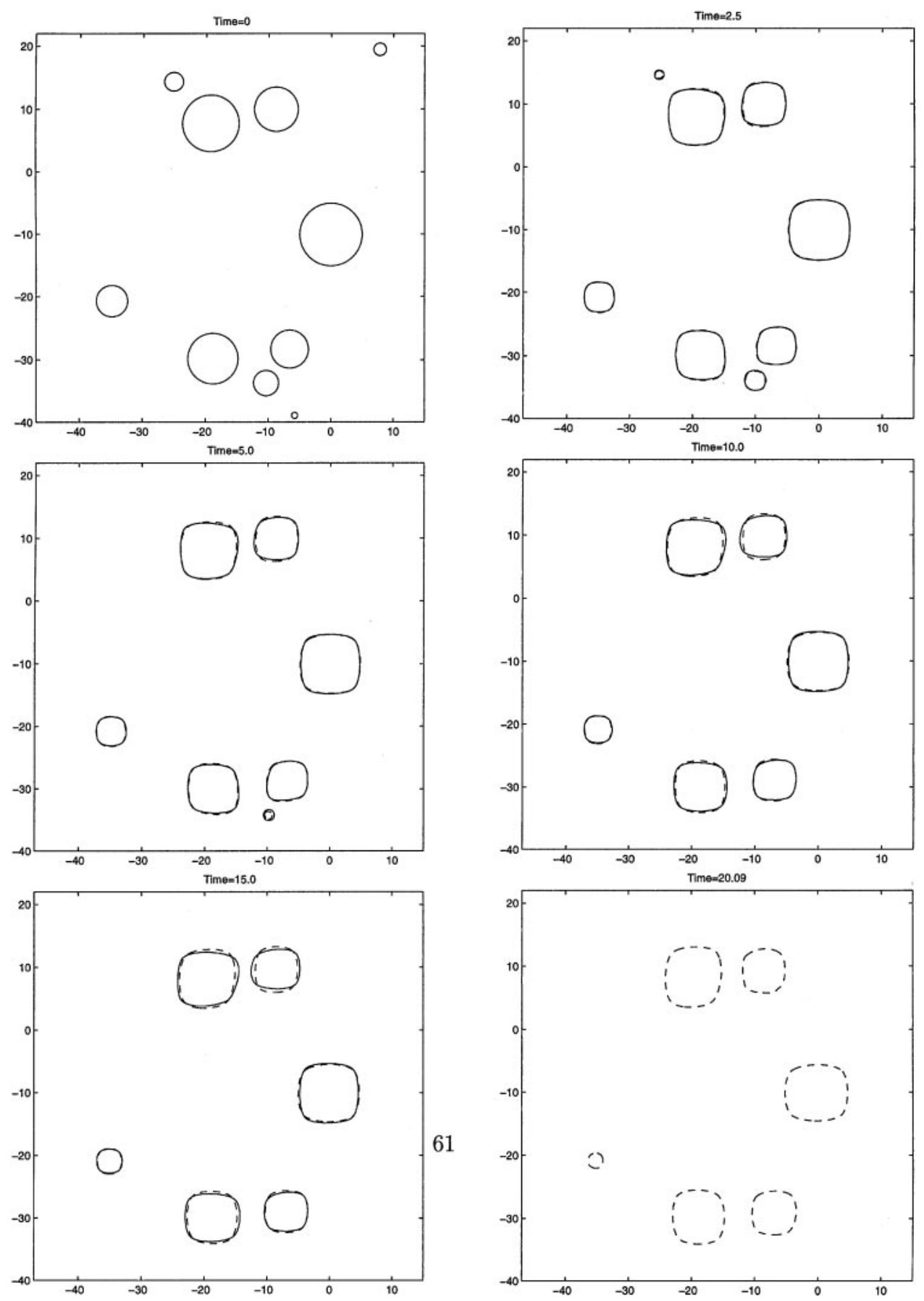

FIG. 19. Evolution of 10 precipitates in a Ni matrix. Solid, $\mathrm{Ni}_{3} \mathrm{Al}$; dashed, $\mathrm{Ni}_{3} \mathrm{Si}, Z=1, \Delta t=5 \times 10^{-4}$, $N=256$ for each precipitate, $t o l=10^{-10}$ for diffusion, and $10^{-5}$ for elasticity. Note that only the $\mathrm{Ni}_{3} \mathrm{Si}_{\text {precipitates }}$ are shown at time $t=20.09$; see text for details. 
Time $=15.77$

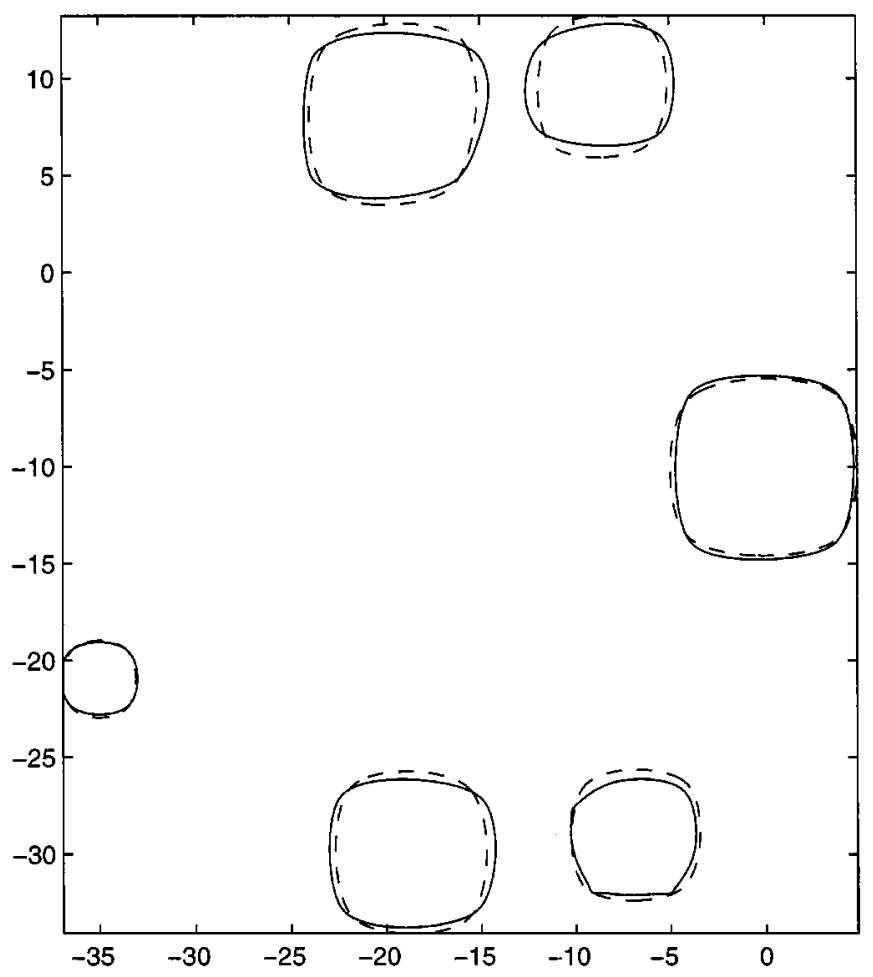

FIG. 20. The six remaining precipitates at $t=15.77$ from the evolution shown in Fig. 19. Solid, $\mathrm{Ni}_{3} \mathrm{Al}$; dashed, $\mathrm{Ni}_{3} \mathrm{Si}$.

upper precipitates attract one another and likely merge. In the $\mathrm{Ni}_{3} \mathrm{Si}$ case, on the other hand, it does not appear that these two precipitates will merge. This is consistent with the results of our two precipitate simulations. In addition, the interacting pairs of $\mathrm{Ni}_{3} \mathrm{Al}$ precipitates tend to be "flatter" than their $\mathrm{Ni}_{3} \mathrm{Si}$ counterparts.

We further observe that the lower two precipitates in the $\mathrm{Ni}_{3} \mathrm{Al}$ case also attract one another. In the process, the lower right precipitate develops very high curvature (note its flat bottom) which ultimately prevents us from continuing the simulation much beyond this time. This is why no $\mathrm{Ni}_{3} \mathrm{Al}$ precipitates are shown at time $t=20.09$. By increasing the numerical resolution of the $\mathrm{Ni}_{3} \mathrm{Al}$ simulation (i.e., using $N=512$ and $N=1024$ ), we are able to continue the simulation slightly further in time. However, much higher local resolutions are needed in order to determine whether the curvature actually develops a singularity at a finite time. In a future work [21], we will use adaptive discretizations to try to answer this question.

\subsection{Orthotropic Elasticity}

As a final case, we now consider examples where the elasticity displays fully orthotropic behavior in two dimensions. That is, we have four independent elastic constants $C_{11}, C_{12}$, $C_{22}$, and $C_{66}$, rather than the three $\left(C_{11}, C_{12}, C_{22}=C_{11}\right.$ and $\left.C_{66}\right)$ needed in cubic elasticity. The example we present relates to an observation of microstructure in geology.

In a set of experiments performed by David Kohlstedt and Mark Zimmerman in the Department of Geology at the University of Minnesota, the mineral olivine, which has an 

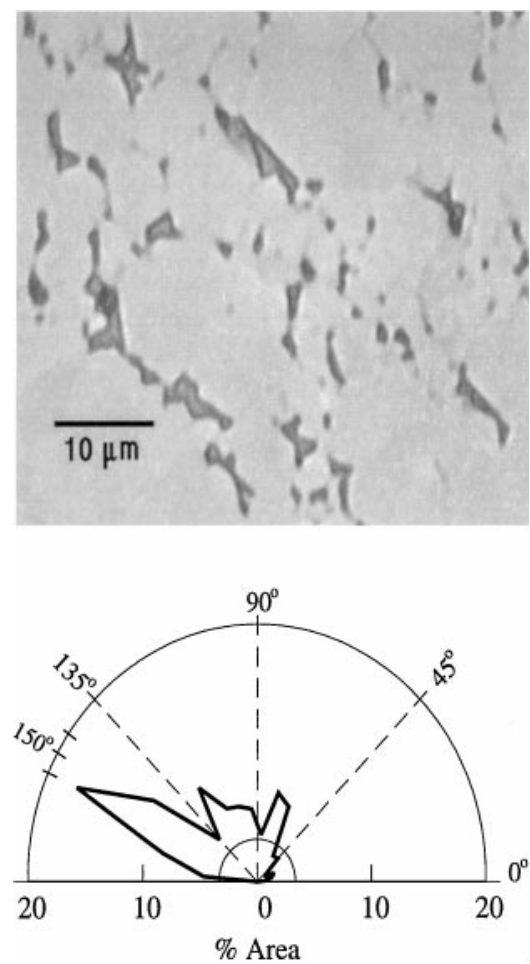

FIG. 21. Top (a), reflected light optical micrographs of the olivine matrix with imbedded melt pockets (dark) for the experiment described in the text. The faint outlines of the grains (surrounded by the melt pockets) can also be observed. The coordinate axes correspond to the crystallographic axes. Bottom (b), polar plot indicating the percentage of melt area in each orientation. Plots courtesy of Kohlstedt and Zimmerman.

orthorhombic crystal structure, was loaded in shear. Melt pockets selectively accumulate with preferred orientations, as indicated in Fig. 21. This preferred orientation becomes more pronounced with increasing shear. The orientation of melt pockets has profound implications for anisotropy in permeability, seismic attenuation, and seismic anisotropy beneath midocean ridges. See [16] for a more detailed explanation of the experiments and description of the results.

Our goal is to use our methods to try to understand the relationship between the melt pocket orientations and the anisotropy of the olivine. We consider the melt pockets to be soft elliptical particles embedded in an orthotropic material with elastic constants appropriate to olivine. The elastic constants used in the simulations are given in Table VII. For the soft precipitate, the ratio of the shear moduli $c_{66},\left(c_{11}-c_{12}\right) / 2$ to bulk modulus $c_{11}+c_{12}$ is approximately equal to 3 . Since real fluids typically have very large such ratios, we checked that increasing the bulk/shear ratio did not significantly alter our results. The simulations we present include applied biaxial compression and shear but no misfit between the particle and matrix phases.

We first consider two interacting particles evolving by diffusion. Figure 22 shows the results of this simulation. The particles translate and elongate to align approximately in the direction of the shear. In addition, they attract one another. This result is consistent with our previous experience [15]. However, this result is not consistent with the experiments of 


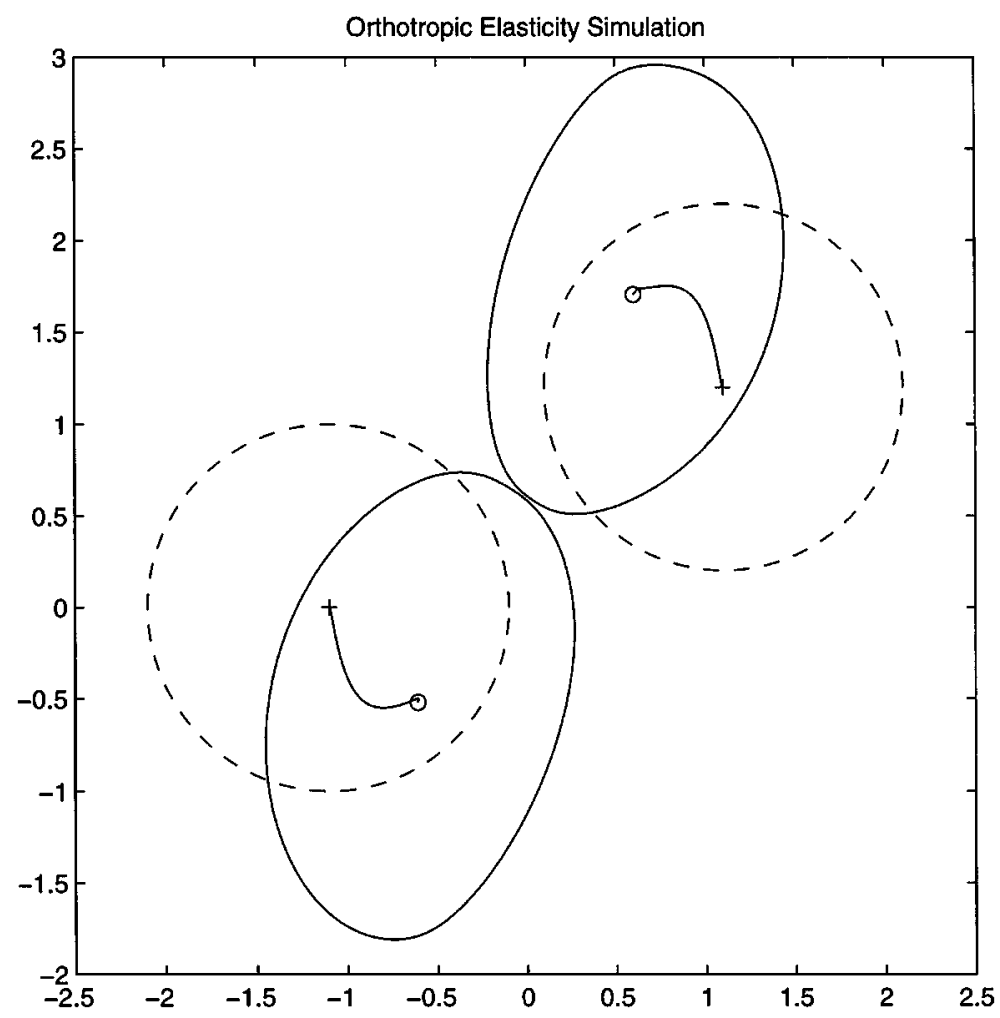

FIG. 22. Two precipitate simulation: Olivine matrix with soft, nearly isotropic precipitates. Isotropic surface tension $\left(\tau_{0}=0\right)$. Dashed circles, initial condition $(T=0)$. Solid curves, precipitates at $T=5.20$. Solid curves with

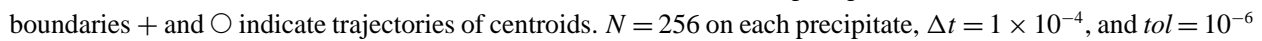
for both diffusion and elasticity.

Kohlstedt and Zimmerman. This is not surprising, as there is no reason to expect that the evolution of the melt pockets is driven by diffusion.

As a next step, we separate the elasticity solver from the diffusional evolution and consider how the average pressure over the precipitate varies with the orientation of the precipitate. This idea is motivated by the possibility that the molten phase seeps into low pressure regions of the olivine crystal (e.g., Darcy's law) [16].

The actual quantity we calculate is the integral of the normal displacement over the precipitate-matrix interface $\left(\bar{u}_{k, k}\right)$. We justify this choice by noting that for an isotropic material, this quantity is proportional to the average pressure with proportionality constant equal to the negative of the bulk modulus of the precipitate. In our case, $\bar{u}_{k, k}$ is not exactly proportional to the pressure in the inclusion, since the elastic constants we use to describe the inclusion phase are not isotropic. However, as the precipitate is nearly isotropic ( $A=0.91)$ and since $\bar{u}_{k, k}$ is easy to compute, we believe $\bar{u}_{k, k}$ is suitable as a trial parameter.

We proceed as follows. We solve the elasticity equations for a single elliptical precipitate with arbitrary orientation under an applied biaxial stress and shear. We determine the orientation of the ellipse that achieves the minimum value of $-\bar{u}_{k, k}$ as a function of the strength of the applied shear. We refer to this as the "minimum pressure ellipse." We then varied the 


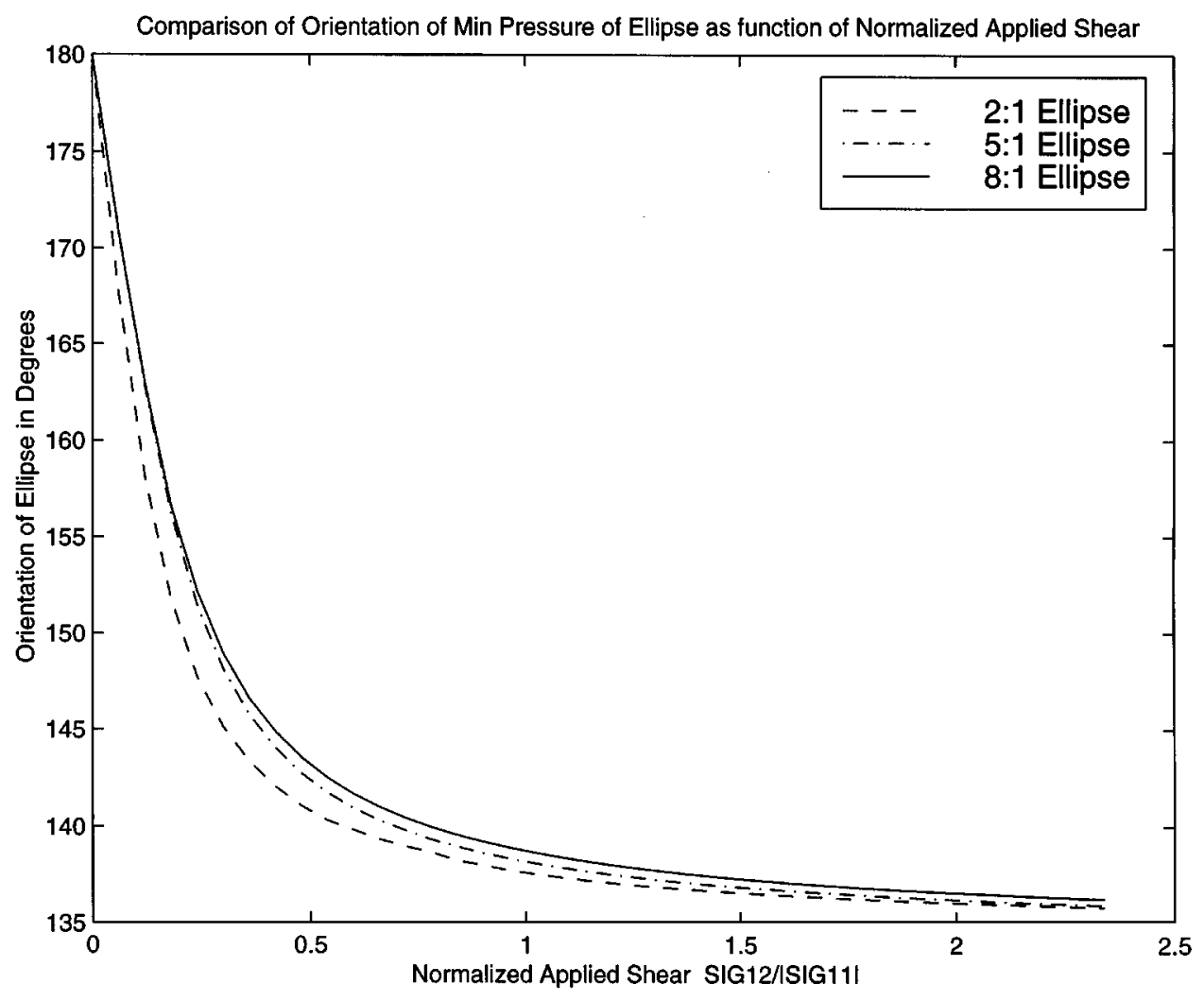

FIG. 23. Static simulation. Orientation of minimum pressure ellipse versus normalized applied shear. Olivine matrix with soft, elliptical nearly isotropic precipitates. Three ellipses with different aspect ratios are considered.

ratio of lengths of the major and minor axes of the ellipse from $2: 1$ to $8: 1$. In the results that follow, we used $N=256$ and the elasticity GMRES tolerance to $10^{-6}$. In Fig. 23, we plot the orientation of the minimum pressure ellipse as a function of applied shear for several different aspect ratios. The orientation angle is measured with respect to the positive $x$-axis with a horizontal ellipse having an orientation of 180 degrees. At zero applied shear, the minimum pressure ellipse is horizontal. As the applied shear is increased, the orientation angle decreases rapidly and seems to asymptote to approximately 135 degrees. In addition, the orientation angle is a non-decreasing function of aspect ratio. Thus, for a given applied shear, larger orientation angles are obtained for ellipses with larger aspect ratios. However, we note that the average pressure per unit area of the minimum pressure ellipse increases with increasing aspect ratio.

To examine the effect of the elastic constants, we modify the constants corresponding to the olivine matrix by either enhancing the orthotropy or by making the matrix more isotropic. In order to enhance the orthotropy $(\bigcirc)$, we set $c_{11}=5.0$ while keeping the other elastic coefficients fixed. In order to make the matrix more isotropic $(+)$, we keep $c_{12}$ and $c_{22}$ fixed and set $c_{11}=3.4$. This gives $A=0.96$. Our results are shown in Fig. 24 for an ellipse with aspect ratio $2: 1$. Analogous results are obtained for other aspect ratios. We observe that increasing the orthotropy increases the angle of the minimum pressure ellipse while increasing the isotropy makes the angle drop faster towards 135 degrees. In fact, if the 


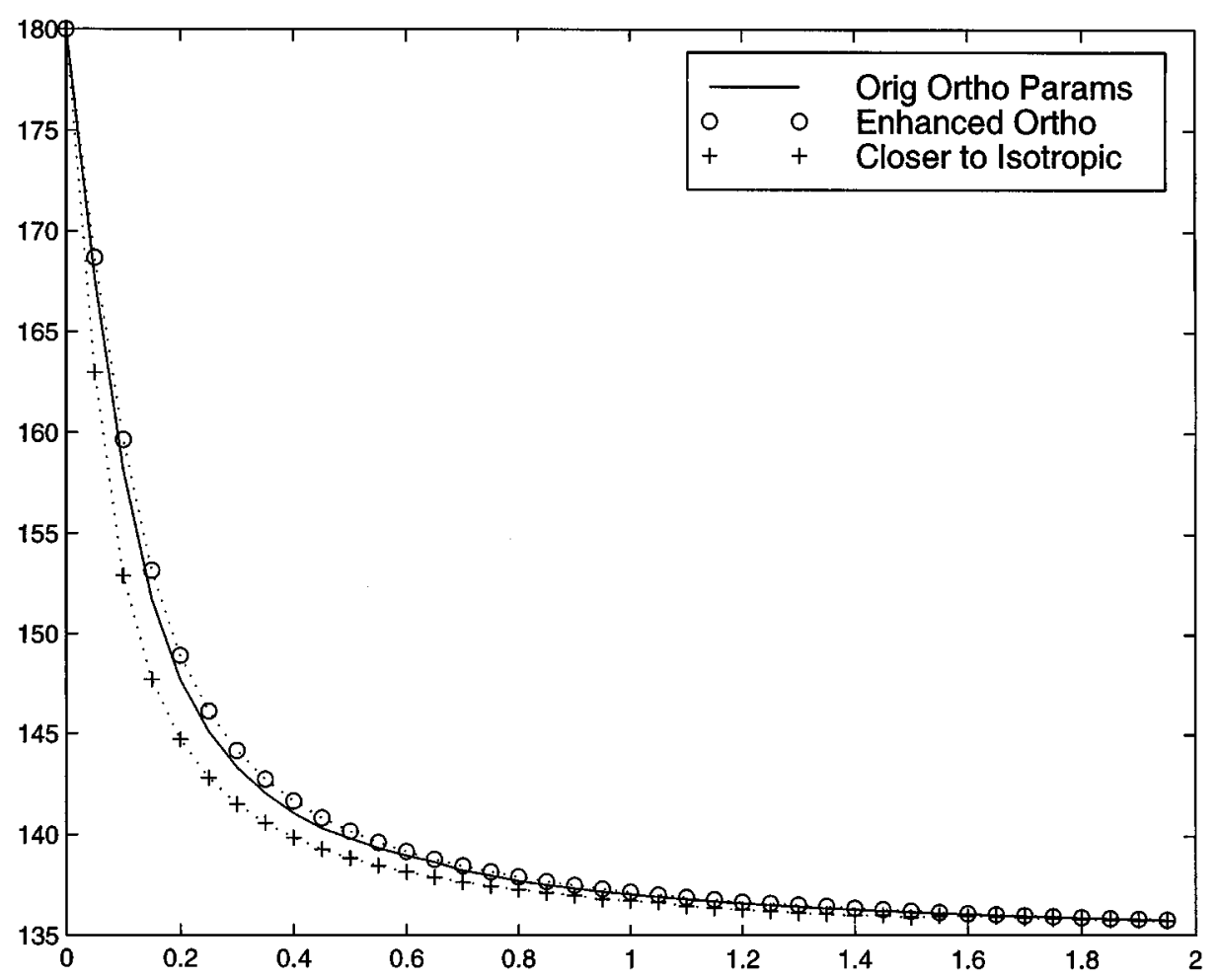

FIG. 24. Static simulation. Orientation of minimum pressure ellipse versus normalized applied shear using different matrix elastic constants. See text for details.

matrix were elastically isotropic, the minimum pressure ellipse is oriented at 135 degrees for all non-zero applied shears.

In the experiment shown in Fig. 21a the normalized applied shear $\left(\sigma_{12} / \sigma_{11}\right)$ is approximately 0.25 . The dominant orientation angle observed in the experiment is $150 \pm 5$ degrees as shown in Fig. 21b. Using the value 0.25 for the normalized shear, we predict orientation angles of approximately 145 degrees for the $2: 1$ ellipse, 148 degrees for the $5: 1$ ellipse, and 149 degrees for the $8: 1$ ellipse. Similar agreement has been found in experiments in which the olivine crystal is loaded in biaxial compression [5]. The fact that the predicted melt pocket orientations are consistent with experiments suggests that the orthotropic character of the olivine may be an important factor in determining the orientation of the melt pockets. Kohlstedt and Zimmerman are currently testing different experimental loading configurations to determine whether the orientation of melt pockets changes with applied shear as predicted in Figs. 23 and 24. We note, however, that there are many other physical factors we do not model, e.g., plastic slip, polycrystallinity, permeability, and fluid flow, which likely play an important role in melt pocket alignment.

\section{APPENDIX: PRECONDITIONING MATRIX FOR ELASTICITY}

For completeness, we present the $4 \times 4$ matrix $\mathcal{L}$ used to precondition the elasticity integral equations in Fourier space. 
For $k \neq 0$, we have

$$
\hat{\mathcal{L}}(k)=\left(\begin{array}{cccc}
1 & \mathrm{i} \operatorname{sgn}(k) D_{21}^{P} & \operatorname{sgn}(k) g_{k} E_{11}^{P} & 0 \\
-\mathrm{i} \operatorname{sgn}(k) D_{12}^{P} & 1 & 0 & \operatorname{sgn}(k) g_{k} E_{22}^{P} \\
1 & -\mathrm{i} \operatorname{sgn}(k) D_{21}^{M} & -\operatorname{sgn}(k) g_{k} E_{11}^{M} & 0 \\
i \operatorname{sgn}(k) D_{12}^{M} & 1 & 0 & -\operatorname{sgn}(k) g_{k} E_{22}^{M}
\end{array}\right),
$$

where $g_{k}=s_{\alpha} / k$. For $k=0$, we obtain

$$
\hat{\mathcal{L}}(0)=\left(\begin{array}{llll}
1 & 0 & 0 & 0 \\
0 & 1 & 0 & 0 \\
1 & 0 & 0 & 0 \\
0 & 1 & 0 & 0
\end{array}\right)
$$

$\hat{\mathcal{L}}^{-1}(k)$ for $k \neq 0$ is analytically constructed using Mathematica. Because $\hat{\mathcal{L}}(0)$ is not invertible, we set the zero modes to be zero.

\section{ACKNOWLEDGMENTS}

It is a pleasure to thank I. Schmidt and P. Voorhees for stimulating and helpful discussions. The authors also acknowledge the support of the Minnesota Supercomputer Institute and the MCS division at Argonne National Laboratory for use of their computational facilities. In addition, P.H.L. was partially supported by the National Science Foundation Grant CMS-9503393. J.S.L. was partially supported by National Science Foundation Grants and the Sloan Foundation. Q.N. was partially supported by the Accelerated Strategic Computing Initiative Center (DOE) and Materials Research Center (NSF) at the University of Chicago. Finally, Q.N. thanks the Institute for Mathematics and Its Applications for its hospitality.

\section{REFERENCES}

1. A. J. Ardell and R. B. Nicholson, On the modulated structure of aged Ni-Al alloys, Acta Metall. 14, 1295 (1966).

2. C. A. Brebbia, J. C. F. Telles, and L. C. Wrobel, Boundary Element Techniques (Springer-Verlag, Berlin, 1984).

3. L. N. Brush and R. F. Sekerka, A numerical study of two-dimensional crystal growth forms in the presence of anisotropic growth kinetics, J. Crystal Growth 96, 419 (1989).

4. W. T. Chen, On an elliptic elastic inclusion in an anisotropic medium, Quart. J. Mech. Appl. Math. 20, 307 (1967).

5. M. J. Daines and D. L. Kohlstedt, Influence of deformation on melt topology in peridotites, J. Geophys. Res. 102, 10,257 (1997).

6. M. Fährman, P. Fratzl, O. Paris, E. Fährmann, and W. C. Johnson, Influence of coherency stress on microstructural evolution in model ni-al-mo alloys, Acta Metall. 43, 1007 (1995).

7. A. E. Green and G. I. Taylor, Stress systems in aeolotropic plates, i, Proc. R. Soc. A 173, 162 (1939).

8. A. Greenbaum, L. Greengard, and G. B. McFadden, Laplace's equation and the Dirichlet-Neumann map in multiply connected domains, J. Comput. Phys. 105, 267 (1993).

9. L. Greengard and V. Rokhlin, A fast algorithm for particle summations, J. Comput. Phys. 73, 325 (1987).

10. M. E. Gurtin and P. W. Voorhees, The continuum mechanics of two-phase elastic solids with mass transport, Proc. R. Soc. London A 440, 323 (1993).

11. C. Herring, Surface tension as a motivation for sintering, in The Physics of Powder Metallurgy, edited by W. E. Kingston (McGraw-Hill, New York, 1951), p. 143. 
12. T. Y. Hou, J. S. Lowengrub, and M. J. Shelley, Removing the stiffness from interfacial flows with surface tension, J. Comput. Phys. 114, 312 (1994).

13. J. K. Tien and S. M. Copley, The effect of uniaxial stress on the periodic morphology of coherent gamma prime precipitates in nickel-base superalloy crystals, Metall. Trans. 2, 215 (1971).

14. W. C. Johnson and J. W. Cahn, Elastically induced shape bifurcations of inclusions, Acta Metall. 32(11), 1925 (1984).

15. H.-J. Jou, P. H. Leo, and J. S. Lowengrub, Microstructural evolution in inhomogeneous elastic media, J. Comput. Phys. 131, 109 (1997).

16. D. L. Kohlstedt and M. E. Zimmerman, Rheology of partially molten mantle rocks, Annu. Rev. Earth Planet Sci. 24, 41 (1996).

17. J. K. Lee, Morphology of coherent particles via a discrete atom method, Materials Sci. Eng. A 238, 1 (1997).

18. J. K. Lee, Coherency strain analysis via a discrete atom method, Scripta Met. 32, 559 (1995).

19. J. K. Lee, A study on coherency strain and precipitate morphology via a discrete atom method, Metall. Trans. A 27, 1449 (1996).

20. P. H. Leo, J. S. Lowengrub, and H.-J. Jou, A diffuse interface model for microstructural evolution in elastically stressed solids, Acta Mater. 46, 2113 (1998).

21. P. H. Leo, J. S. Lowengrub, and Q. Nie, The long time evolution of microstructures in elastic media, in preparation.

22. P. H. Leo and R. F. Sekerka, The effect of surface stress on crystal-melt and crystal-crystal equilibrium, Acta Metall. 37, 3119 (1989).

23. D. Y. Li and L. Q. Chen, Selective variant growth of coherent ti ${ }_{11} \mathrm{ni}_{14}$ precipitates in a tini alloy under applied stress, Acta Mater. 45, 471 (1997).

24. D. Y. Li and L. Q. Chen, Shape evolution and splitting of coherent particles under applied stress, Acta Mater. 47, 247 (1998).

25. A. Maheshwari and A. J. Ardell, Elastic interactions and their effect on $\gamma^{\prime}$ precipitate shapes in aged dilute nial alloys, Scripta Metall. 26, 347 (1992).

26. A. Maheshwari and A. J. Ardell, Morphological evolution of coherent misfitting particles in anisotropic elastic media, Phys. Rev. Lett. 70, 2305 (1993).

27. M. McCormack, A. G. Khachaturyan, and J. W. Morris, A two-dimensional analysis of the evolution of coherent precipitates in elastic media, Acta Metall. 40, 325 (1992).

28. T. Miyazaki, K. Hakamura, and H. Mori, Experimental and theoretical investigations on morphological changes of gamma' precipitates in Ni-Al single crystals during uniaxial stress-annealing, J. Mater. Sci. 14, 1827 (1979).

29. Qing Nie, Topics in the Motion of Bubbles in Incompressible Liquids, Ph.D. thesis, The Ohio State University, 1995.

30. H. Nishimori and A. Onuki, Pattern formation in phase-separating alloys with cubic symmetry, Phys. Rev. B 42, 980 (1990).

31. D. Orlikowski, C. Sagui, A. Somoza, and C. Roland, Large-scale simulations of phase separation of elastically coherent binary alloy systems, Phys. Rev. B 59, 8646 (1999).

32. S. V. Prikhodko, J. D. Carnes, D. G. Isaak, and A. J. Ardell, Elastic constants of a ni-12.69 at, Scripta Mater. 38, 67 (1997).

33. M. Reed and B. Simon, Functional analysis, in Methods of Modern Mathematical Physics (Academic Press, New York, 1980), Vol. 1.

34. F. J. Rizzo and D. J. Shippy, A method for stress determination in plane anisotropic bodies, J. Comp. Mater. 4, 36 (1970).

35. Y. Saad and M. R. Schultz, GMRES: A generalized minimum residual method for solving nonsymmetric linear systems, SIAM J. Sci. Statist. Comput. 7, 856 (1986).

36. C. Sagui, D. Orlikowski, A. Somoza, and C. Roland, Three dimensional simulations of ostwald ripening with elastic effects, Phys. Rev. E 58:R4092 (1998). 
37. I. Schmidt and D. Gross, A strategy for determining the equilibrium shape of an inclusion, Arch. Mech. 47, 379 (1995).

38. I. Schmidt and D. Gross, The equilibrium shape of an elastically inhomogeneous inclusion, J. Mech. Phys. Solids 45, 1521 (1997).

39. I. Schmidt, R. Mueller, and D. Gross, The effect of elastic inhomogeneity on equilibrium and stability of a two particle morphology, Mech. Mater. 30, 181 (1998).

40. A. Sidi and M. Israeli, Quadrature methods for periodic singular and weakly singular Fredholm integral equations, J. Sci. Comp. 3, 201 (1988).

41. C. H. Su and P. W. Voorhees, The dynamics of precipitate evolution in elastically stressed solids. i. Inverse coarsening, Acta Mater. 44, 1987 (1996).

42. C. H. Su and P. W. Voorhees, The dynamics of precipitate evolution in elastically stressed solids. ii. Particle alignment, Acta Mater. 44, 2001 (1996).

43. M. E. Thompson, C. S. Su, and P. W. Voorhees, The equilibrium shape of a misfitting precipitate, Acta Metall. 42, 2107 (1994).

44. Y. Wang, L. Chen, and A. G. Khachaturyan, Computer simulation of microstructure evolution in coherent solids, in Solid-Solid Phase Transformations, edited by W. C. Johnson, J. M. Howe, D. E. Laughlin, and W. A. Soffa (The Minerals, Metals and Materials Society, Pennsylvania, 1994), p. 245.

45. Y. Wang and A. G. Khachaturyan, Shape instability during precipitate growth in coherent solids, Acta Metall. 43, 1837 (1995).

46. Y. S. Yoo, D. Y. Yoon, and M. F. Henry, The effect of elastic misfit strain on the morphological evolution of $\gamma^{\prime}$-precipitates in a model Ni-base superalloy, Metals Mater. 1, 47 (1995). 